\title{
VirTex: Learning Visual Representations from Textual Annotations
}

\author{
Karan Desai Justin Johnson \\ University of Michigan \\ $\{$ kdexd, justincj\}@umich.edu
}

\begin{abstract}
The de-facto approach to many vision tasks is to start from pretrained visual representations, typically learned via supervised training on ImageNet. Recent methods have explored unsupervised pretraining to scale to vast quantities of unlabeled images. In contrast, we aim to learn high-quality visual representations from fewer images. To this end we revisit supervised pretraining, and seek dataefficient alternatives to classification-based pretraining. We propose VirTex - a pretraining approach using semantically dense captions to learn visual representations. We train convolutional networks from scratch on COCO Captions, and transfer them to downstream recognition tasks including image classification, object detection, and instance segmentation. On all tasks, VirTex yields features that match or exceed those learned on ImageNet - supervised or unsupervised - despite using up to ten times fewer images.
\end{abstract}

\section{Introduction}

The prevailing paradigm for learning visual representations is first to pretrain a convolutional network $[1,2]$ to perform image classification on ImageNet [3, 4], then transfer the learned features to downstream tasks [5, 6]. This approach has been wildly successful, and has led to significant advances on a wide variety of computer vision problems such as object detection [7], semantic [8] and instance [9] segmentation, image captioning [10-12], and visual question answering $[13,14]$. Despite its practical success, this approach is expensive to scale since the pretraining step relies on images annotated by human workers.

For this reason, there has been increasing interest in unsupervised pretraining methods that use unlabeled images to learn visual representations which are then transferred to downstream tasks [15-21]. Some recent approaches have begun to match or exceed supervised pretraining on ImageNet [22-26], and have been scaled to hundreds of millions [22, 25, 27, 28] or billions [24] of images.

Continuing to scale unsupervised pretraining to everlarger sets of unlabeled images is an important scientific

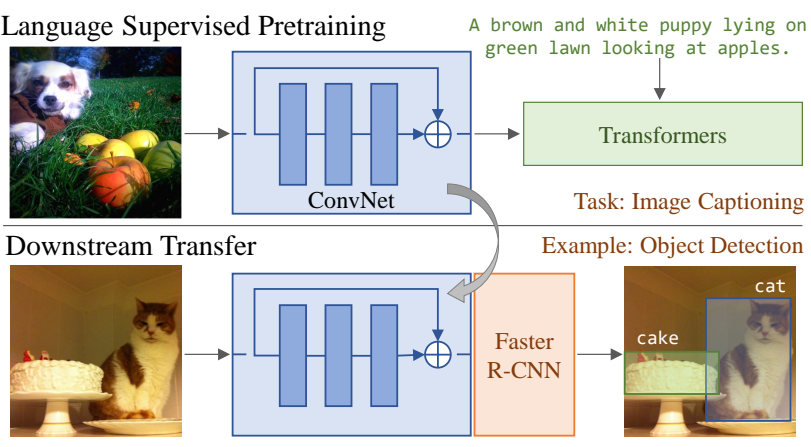

Figure 1: Learning visual features from language: First, we jointly train a ConvNet and Transformers using imagecaption pairs, for the task of image captioning (top). Then, we transfer the learned ConvNet to several downstream vision tasks, for example object detection (bottom).

goal. But we may also ask whether there are alternate ways of pretraining that learn high-quality visual representations with fewer images. To do so, we revisit supervised pretraining and seek an alternative to traditional classification pretraining that uses each image more efficiently.

In this paper we present an approach for learning Visual representations from Textual annotations (VirTex). Our approach is straightforward: first, we jointly train a ConvNet and Transformer [29] from scratch to generate natural language captions for images. Then, we transfer the learned features to downstream visual recognition tasks (Figure 1).

We believe that using language supervision is appealing due to its semantic density. Figure 2 compares different pretraining tasks for learning visual representations. Captions provide a semantically denser learning signal than unsupervised contrastive methods and supervised classification. Hence, we expect that using textual features to learn visual features may require fewer images than other approaches.

Another benefit of textual annotations is simplified data collection. To collect classification labels, typically human experts first build an ontology of categories [3, 4, 30, 31], then complex crowdsourcing pipelines are used to elicit labels from non-expert users [32,33]. In contrast, natural language descriptions do not require an explicit ontology and can easily be written by non-expert workers, leading to a 


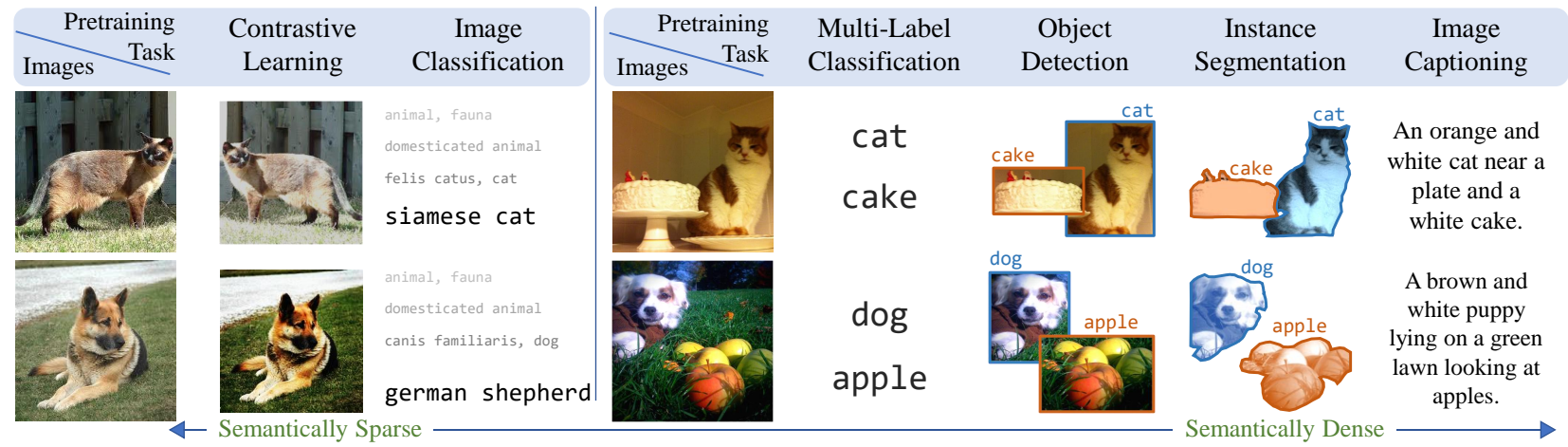

Figure 2: Comparison of pretraining tasks for learning visual representations: Contrastive self-supervised learning methods provide a semantically sparse learning signal, encouraging different transforms of an image to have similar features. Image classification pairs an image with a single semantic concept, providing moderate semantic density. Multi-label classification, object detection, and instance segmentation increase semantic density by labeling and localizing multiple objects. Captions describe multiple objects, their attributes, relationships, and actions, giving a semantically dense learning signal. In this work, we aim to leverage this semantic density of captions to learn visual representations in a data-efficient manner.

simplified data collection pipeline [34-36]. Large quantities of weakly aligned images and text can also be obtained from internet images [37-39].

Our main contribution is to show that natural language can provide supervision for learning transferable visual representations with better data-efficiency than other approaches. We train models from scratch on the COCO Captions dataset [36], and evaluate the learned features on downstream tasks including image classification, object detection, instance segmentation, and low-shot recognition. On all tasks, VirTex matches or exceeds the performance of existing methods for supervised or unsupervised pretraining on ImageNet, despite using up to $10 \times$ fewer images. Our code and pretrained models are available at https://github.com/kdexd/virtex

\section{Related Work}

Our work is related to recent efforts to move beyond supervised pretraining on ImageNet using alternate data sources or pretraining tasks.

Weakly Supervised Learning scales beyond supervised pretraining with a quantity over quality approach, and learns on large numbers of images with noisy labels from web services. Li et al. [40] trains visual N-gram models on the YFCC-100M dataset [41], that provides 100M Flickr images with user-provided tags. Recent works [42-44] also use JFT-300M [42] dataset, curated by automatic labeling of images from web signals using Google's internal tooling. Weakly-supervised learning has also been studied on up to 3.5B Instagram images, using hashtags as labels [45, 46]. These approaches learn visual representations with large quantities of images with low-quality labels; in contrast we focus on using fewer images with high-quality annotations. Self-Supervised Learning focuses on learning visual rep- resentations by solving pretext tasks defined on unlabeled images. Early works on self-supervised learning proposed hand-crafted pretext tasks, such as context prediction [15], colorization [17, 18], solving jigsaw puzzles [47], predicting rotation [19], inpainting [16], clustering [27], and generative modeling [48]. Recent works are based on contrastive learning $[49,50]$, encouraging similarity between image features under different random transformations on single input image [24-26, 51, 52]. Other approaches use contrastive losses based on context prediction [20,23], mutual information maximization [21, 53, 54], predicting masked regions [55], and clustering [56-58].

These methods lack semantic understanding as they rely on low-level visual cues (color, texture), whereas we leverage textual annotations for semantic understanding. Unlike these methods, our approach can leverage additional metadata such as text, when scaled to internet images [37-39].

Vision-and-Language Pretraining attempts to learn joint representations of image-text paired data that can be transferred to multimodal downstream tasks such as visual question answering [13, 14, 59, 60], visual reasoning [61, 62], referring expressions [63], and language-based image retrieval [35]. Inspired by the success of BERT [64] in NLP, several recent methods use Transformers [29] to learn transferable joint representations of images and text [65-72].

These methods employ complex pretraining pipelines: they typically (1) start from an ImageNet-pretrained CNN; (2) extract region features using an object detector finetuned on Visual Genome [73], following [74]; (3) optionally start from a pretrained language model, such as BERT [64]; (4) combine the models from (2) and (3), and train a multimodal transformer on Conceptual Captions [37]; (5) fine-tune the model from (4) on the downstream task. In this pipeline, all vision-and-language tasks are downstream from the initial visual representations learned on ImageNet. 
In contrast, we pretrain via image captioning, and put vision tasks downstream from vision-and-language pretraining.

Concurrent Work: Our work is closest to Sariyildiz et al. [75] on learning visual representations from captions via image conditioned masked language modeling, with one major difference - we train our entire model from scratch, whereas they rely on pretrained BERT for textual features. Moreover, we evaluate on additional downstream tasks like object detection and instance segmentation. Our work is also closely related to Stroud et al. [76] on learning video representations using paired textual metadata, however they solely operate and evaluate their method on video tasks.

\section{Method}

Given a dataset of image-caption pairs, our goal is to learn visual representations that can be transferred to downstream visual recognition tasks. As shown in Figure 2, captions carry rich semantic information about images, including the presence of objects (cat, plate, cake); attributes of objects (orange and white cat); spatial arrangement of objects (cat near a plate); and their actions (looking at apples). Learned visual representations that capture such rich semantics should be useful for many downstream vision tasks.

To this end, we train image captioning models to predict captions from images. As shown in Figure 3, our model has two components: a visual backbone and a textual head. The visual backbone extracts visual features from an input image $I$. The textual head accepts these features and predicts a caption $C=\left(c_{0}, c_{1}, \ldots, c_{T}, c_{T+1}\right)$ token by token, where $c_{0}=$ [SOS] and $c_{T+1}=$ [EOS $]$ are fixed special tokens indicating the start and end of sentence. The textual head performs bidirectional captioning (bicaptioning): it comprises a forward model that predicts tokens left-to-right, and a backward model that predicts right-to-left. All model components are randomly initialized, and jointly trained to maximize the log-likelihood of the correct caption tokens

$$
\begin{array}{r}
\mathcal{L}(\theta, \phi)=\sum_{t=1}^{T+1} \log \left(p\left(c_{t} \mid c_{0: t-1}, I ; \phi_{f}, \theta\right)\right) \\
+\sum_{t=0}^{T} \log \left(p\left(c_{t} \mid c_{t+1: T+1}, I ; \phi_{b}, \theta\right)\right)
\end{array}
$$

where $\theta, \phi_{f}$, and $\phi_{b}$ are the parameters of the visual backbone, forward, and backward models respectively. After training, we discard the textual head and transfer the visual backbone to downstream visual recognition tasks.

Language Modeling: Our choice of pretraining task is image captioning [10-12] - a well-studied vision-andlanguage task, so far kept downstream from vision-based pretraining. We draw inspiration from recent work in NLP using language modeling as a pretraining task to learn transferable text representations. This involves training mas- sive language models - either unidirectional [77] or bidirectional [78-81], for predicting tokens one by one. However, following BERT [64], many large-scale models [82, 83] instead use masked language models (MLMs): some tokens are randomly masked and are predicted by the model.

We performed preliminary experiments with MLMs, but like $[64,84]$ we observed that MLMs converge more slowly than directional models. We note that MLMs have poor sample efficiency, as they only predict a subset of tokens for each caption, while directional models predict all tokens. Due to computational constraints, we focus on directional models and leave MLMs to future work.

Visual Backbone: The visual backbone is a convolutional network which computes visual features of images. It inputs raw image pixels, and outputs a spatial grid of image features. During pretraining, these features are used to predict captions. In downstream tasks, we either train linear models on features extracted from the visual backbone, or fine-tune the visual backbone end-to-end.

In principle we could use any convolutional network architecture for the visual backbone. In our experiments we use a standard ResNet-50 [2] as the visual backbone to facilitate comparison with our baseline methods (Section 4). It accepts a $224 \times 224$ image and produces a $7 \times 7$ grid of 2048-dimensional features after the final convolutional layer. During pretraining, we apply a linear projection layer to the visual features before passing them to the textual head to facilitate decoder attention over visual features. This projection layer is not used in downstream tasks.

Textual Head: The textual head receives features from the visual backbone and predicts captions for images. It provides a learning signal to the visual backbone during pretraining. Our overall goal is not to predict high-quality captions, but instead to learn transferable visual features.

The textual head comprises two identical language models which predict captions in forward and backward directions respectively. Following recent advances in language modeling, we use Transformers [29], which use multiheaded self-attention both to propagate information along the sequence of caption tokens, as well as to fuse visual and textual features. We closely follow the transformer decoder architecture from [29], but use GELU [85] rather than ReLU, following [64, 79]. We briefly review the architecture here; refer to [29] for a more complete description.

During training, the forward model receives two inputs: image features from the visual backbone, and a caption describing the image. Image features are a matrix of shape $N_{I} \times D_{I}$ giving a $D_{I}$-dimensional vector for each of the $N_{I}=7 \times 7$ positions in the final layer of the visual backbone. As described earlier, the caption $C=$ $\left(c_{0}, c_{1}, \ldots, c_{T}, c_{T+1}\right)$ is a sequence of $T+2$ tokens, with $c_{0}=$ [SOS] and $c_{T+1}=$ [EOS]. It is trained to predict $C_{1: T+1}$ token-by-token, starting with $c_{0}$. The prediction $c_{t}$ 


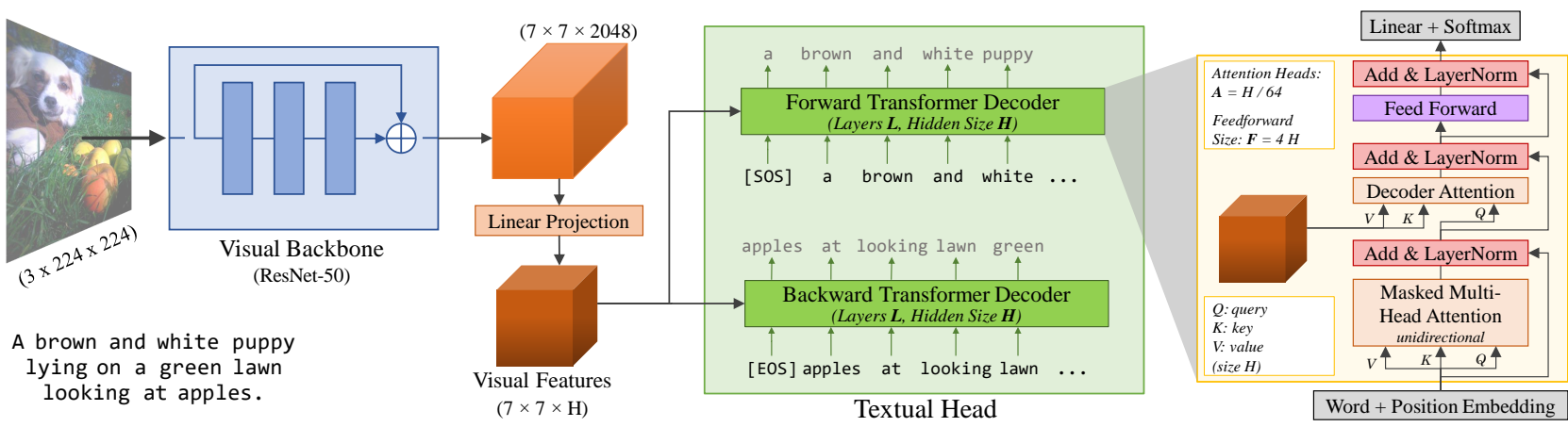

Figure 3: VirTex pretraining setup: Our model consists of a visual backbone (ResNet-50), and a textual head (two unidirectional Transformers). The visual backbone extracts image features, and textual head predicts captions via bidirectional language modeling (bicaptioning). The Transformers perform masked multiheaded self-attention over caption features, and multiheaded attention over image features. Our model is trained end-to-end from scratch. After pretraining, the visual backbone is transferred to downstream visual recognition tasks.

is causal - it only depends on past predictions $c_{0: t-1}$ and visual features. The backward model is similar; it operates right-to-left - trained to predict $C_{T: 0}$, given $c_{T+1}$.

First, we convert the tokens of $C$ to vectors via learned token and positional embeddings, followed by elementwise sum, layer normalization [86] and dropout [87]. Next, we process these vectors through a sequence of Transformer layers. As shown in Figure 3, each layer performs masked multiheaded self-attention over token vectors, multiheaded attention between token vectors and image vectors, and applies a two-layer fully-connected network to each vector. These three operations are each followed by dropout, wrapped in a residual connection, and followed by layer normalization. Token vectors interact only through selfattention; the masking in this operation maintains causal structure of the final predictions. After the last Transformer layer, we apply a linear layer to each vector to predict unnormalized log-probabilities over the token vocabulary.

The forward and backward models consist of independent Transformer layers. However they share the same token embedding matrix (similar to [77]) which is also reused at the output layers of each model (similar to [88, 89]).

Model Size: Several architectural hyperparameters control the size of our textual head. We can control the width of each Transformer layer by varying its hidden size $H$, the number of attention heads $A$ used in multiheaded attention, and the feedforward size $F$ of the fully-connected network. We follow [64] and always set $A=H / 64$ and $F=4 H$; this allows us to control the width of our textual head by varying $H$. We can also control the depth of our textual head by varying the number of transformer layers $L$.

Tokenization: We tokenize captions with SentencePiece [90] using the BPE algorithm [91]. Prior to tokenization we lowercase and strip accents from captions. We build a vocabulary of $10 \mathrm{~K}$ tokens, including boundary ([SOS], [EOS]) and out-of-vocab ([UNK]) tokens. Follow- ing $[79,80]$ we restrict subword merges between letters and punctuation to prevent redundant tokens such as dog? and dog!. Compared to basic tokenization schemes often used for image captioning that split on whitespace [10, 11], BPE makes fewer linguistic assumptions, exploits subword information, and results in fewer out-of-vocab tokens.

Training Details: We train on the train2017 split of the COCO Captions dataset [36], which provides $118 \mathrm{~K}$ images with five captions each. During training we apply standard data augmentation: we randomly crop to $20-100 \%$ of the original image size, apply color jitter (brightness, contrast, saturation, hue), and normalize using the ImageNet mean color. We also apply random horizontal flips, also interchanging the words 'left' and 'right' in the caption.

We train using SGD with momentum $0.9[92,93]$ and weight decay $10^{-4}$ wrapped in LookAhead [94] with $\alpha=$ 0.5 and 5 steps. Following [64], we do not apply weight decay to layer normalization and bias parameters in Transformers. We perform distributed training across 8 GPUs with batch normalization [95] per GPU, following [22]. We train with a batch size of 256 images ( 32 per GPU) for $500 \mathrm{~K}$ iterations $(\approx 1080$ epochs). We use linear learning rate warmup [22] for the first $10 \mathrm{~K}$ iterations followed by cosine decay [96] to zero. We found that the visual backbone required a higher LR than the textual head for faster convergence. The visual backbone uses a max LR of $2 \times 10^{-1}$; the textual head uses $10^{-3}$. We implement our models using PyTorch [97] with native automatic mixed-precision [98].

We observe that performance on image captioning has a positive but imprecise correlation with performance on downstream visual recognition tasks (Refer Appendix A.4). We thus perform early stopping based on the performance of our visual backbone on downstream PASCAL VOC [99] linear classification (see Section 4.1) since it is fast to evaluate and correlates well with our other downstream tasks. 


\begin{tabular}{lcccl}
\hline Method & Annotations & Cost (hours) $\dagger$ & VOC07 & IN-1k \\
\hline MoCo-COCO & self-sup. & - & 63.3 & 41.1 \\
Multi-label Clf. & labels & $11.1 \mathrm{~K}[30]$ & 86.2 & 46.2 \\
Instance Segmentation & masks & $30.0 \mathrm{~K}[30]$ & 82.3 & 51.0 \\
\hline VirTex (1 caption) & captions & $1.3 \mathrm{~K}[100]$ & 84.2 & 50.2 \\
VirTex (5 caption) & captions & $6.5 \mathrm{~K}[100]$ & $\mathbf{8 8 . 7}$ & $\mathbf{5 3 . 8}$ \\
\hline
\end{tabular}

Table 1: Annotation Cost Efficiency: We compare downstream performance of various pretraining methods on COCO. VirTex outperforms all other methods trained on the same set of images with best performance vs. cost tradeoff. †: For COCO train2017 split, see Appendix A.1 for more details.

\section{Experiments}

In our experiments, we aim to demonstrate the effectiveness of learning visual features via natural language supervision. As described in Section 3, we jointly train a VirTex model from scratch on the COCO Captions [36] dataset. Here, we evaluate the features learned by visual backbone on six downstream vision tasks. We select these tasks based on two common mechanisms for transfer learning: where the visual backbone is either used as (a) frozen feature extractor, or (b) weight initialization for fine-tuning.

\subsection{Image Classification with Linear Models}

Our first set of evaluations involve training linear models on frozen visual backbones - we compare VirTex with various pretraining methods to test our two hypotheses:

1. Learning visual features via captions is cheaper than using other types of annotations, like labels and masks.

2. Using semantically dense captions helps with learning effective visual features using fewer training images.

We evaluate on two datasets: PASCAL VOC [99] and ImageNet-1k [4]. We choose these tasks based on their simplicity and evaluation speed. We briefly describe the setup here. Refer Appendix A.1 for more details.

PASCAL VOC: We follow same protocol as SwAV [58] (highly similar to $[22,25]$ ); we train on VOC07 trainval split (9K images, 20 classes) and report mAP on test split. We train per-class SVMs on 2048-dimensional global average pooled features extracted from the last layer of the visual backbone. For each class, we train SVMs for cost values $C \in\{0.01,0.1,1,10\}$ and select best $C$ by 3 -fold crossvalidation. Other SVM hyperparameters are same as [22].

ImageNet-1k: We follow similar protocol as MoCo [24] and SwAV [58]: we train on the ILSVRC 2012 train split and report top-1 accuracy on val split. We train a linear classifier (fully connected layer + softmax) on 2048dimensional global average pooled features extracted from the last layer of the visual backbone. We train with batch size 256 distributed across 8 GPUs for 100 epochs. We use SGD with momentum 0.9 and weight decay 0 . We set the initial LR to 0.3 and decay it to zero by cosine schedule.

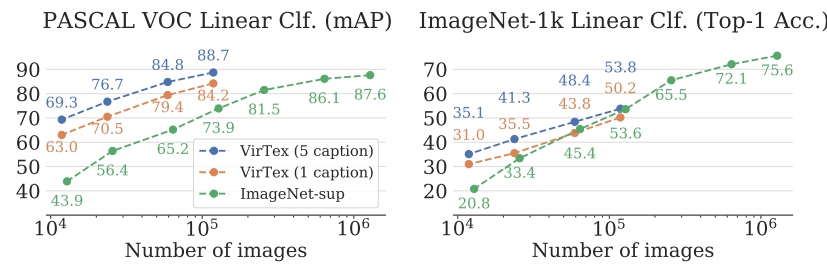

Figure 4: Data Efficiency: We compare VirTex and INsup models trained using varying amounts of images. VirTex closely matches or significantly outperforms IN-sup on downstream tasks despite using $10 \times$ fewer images. $I N-1 k$ : Models using $\leq 10^{5}$ images are mean of 5 trials, std dev. $\leq 1.0$.

Annotation Cost Efficiency: We believe that using captions is appealing due to a simple and cost-efficient collection pipeline. Here, we test our first hypothesis by comparing various pretraining methods on $\mathrm{COCO}$, each drawing supervision from different annotation types (Figure 2):

- MoCo-COCO (self-supervised): We train a MoCo-v1 model on COCO images with default hyperparameters.

- Multi-label Classification (labels): We use COCO object detection annotations (80 classes), and train a ResNet-50 backbone to predict a $K$-hot vector with values $1 / K$ with a KL-divergence loss, similar to [45].

- Instance Segmentation (masks): We use a pretrained Mask R-CNN from Detectron2 model zoo [101], and extract its ResNet-50 backbone for downstream tasks. This model is trained from scratch on COCO, following [102].

- VirTex (captions): We train a VirTex model on COCO Captions, with ResNet-50 visual backbone and $L=$ $1, H=2048$ textual head. Note that COCO Captions provides five captions per image, which effectively increases image-caption pairs by five-fold. Hence for a fair comparison, we also train an additional VirTex model using only one randomly selected caption per image.

Results are shown in Table 1. We also compare annotation costs in terms of worker hours. For labels and masks, we use estimates reported by COCO [30]. For captions, we estimate the cost based on nocaps [100] ${ }^{1}$, that follows a similar data collection protocol as COCO. We observe that VirTex outperforms all methods, and has the best performance vs. cost tradeoff, indicating that learning visual features using captions is more cost-efficient than labels or masks.

Data Efficiency: We believe that the semantic density of captions should allow VirTex to learn effective visual features from fewer images than other methods. To test our hypothesis, we compare VirTex and ImageNet-supervised models (IN-sup) trained using varying amount of images from COCO Captions and ImageNet-1k respectively.

We train 4 VirTex models using $\{10,20,50,100\} \%$ of COCO Captions (118K images) and 7 ResNet-50 models using $\{1,2,5,10,20,50,100\} \%$ of ImageNet-1k (1.28M

\footnotetext{
${ }^{1}$ We could not find estimates for COCO Captions in existing literature.
} 


\begin{tabular}{|c|c|c|c|c|}
\hline Method & Pretrain Images & Annotations & VOC07 & IN-1k \\
\hline MoCo-IN v1 [24] & $1.28 \mathrm{M}$ & self-sup. & 79.4 & 60.8 \\
\hline PCL v1 [57] & $1.28 \mathrm{M}$ & self-sup. & 83.1 & 61.5 \\
\hline SwAV (200 ep.) [58] & $1.28 \mathrm{M}$ & self-sup. & 87.9 & 72.7 \\
\hline ICMLM $_{a t t-f c}[75] \dagger$ & $118 \mathrm{~K}$ & captions & 87.5 & 47.9 \\
\hline VirTex & $118 \mathrm{~K}$ & captions & 88.7 & 53.8 \\
\hline
\end{tabular}

Table 2: Comparison with other methods: We compare downstream performance of VirTex with recent SSL methods and concurrent work. $\dagger$ : Uses pretrained BERT-base.

images). Similar to prior experiments, we also train 4 VirTex models using one randomly selected caption per image. All VirTex models use $L=1, H=2048$ textual heads.

We show results in Figure 4. On VOC07, VirTex-100\% outperforms IN-sup- $100 \%$ (mAP 88.7 vs 87.6), despite using $10 \times$ fewer images $(118 \mathrm{~K}$ vs. $1.28 \mathrm{M})$. When using similar amount of images, VirTex consistently outperforms INsup (blue, orange vs green), indicating superior data efficiency of VirTex. We also observe that given the same number of captions for training, it is better to spread them over more images - VirTex-50\% (1 caption) significantly outperforms VirTex-10\% (5 captions) (mAP 79.4 vs 69.3).

Comparison with IN-sup on ImageNet-1k classification is unfair for VirTex, since IN-sup models are trained for the downstream task, using the downstream dataset. Even so, VirTex-100\% outperforms IN-sup- $10 \%$ (53.8 vs. 53.6, $118 \mathrm{~K}$ vs. $128 \mathrm{~K}$ images), and consistently outperforms it when both methods use fewer than $100 \mathrm{~K}$ images.

Comparison with other methods: Here, we compare VirTex with recent pretraining methods that have demonstrated competitive performance on downstream tasks.

- Self-supervised pretraining: We choose three recent methods based on their availability and compatibility with our evaluation setup - MoCo [24], PCL [57], and SwAV [58]. We choose models trained with a similar compute budget as ours (8 GPUs, 200 ImageNet epochs).

- ICMLM (Concurrent Work): We adapt numbers from Sariyildiz et al. [75]; evaluation may slightly differ. This model uses pretrained BERT [64] for textual features.

- Note on vision-and-language pretraining: Since we use captions, we also consider methods that learn multimodal representations for downstream vision-andlanguage tasks [65-72]). As described in Section 2, all these methods use an object detector trained on Visual Genome [73] (with ImageNet-pretrained backbone) to extract visual features, made available by [74]. These features are kept frozen, and do not learn from any textual supervision at all. Our comparison with ImageNetsupervised models subsumes this family of models.

Results are shown in Table 2. VirTex outperforms all methods on VOC07, despite being trained with much fewer images. On ImageNet-1k, comparison between self-

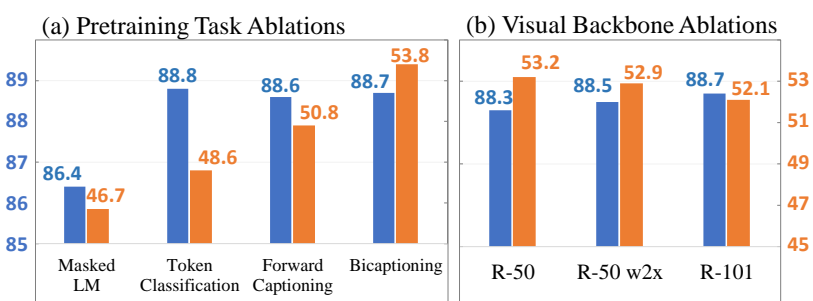

(c) Transformer Size Ablations

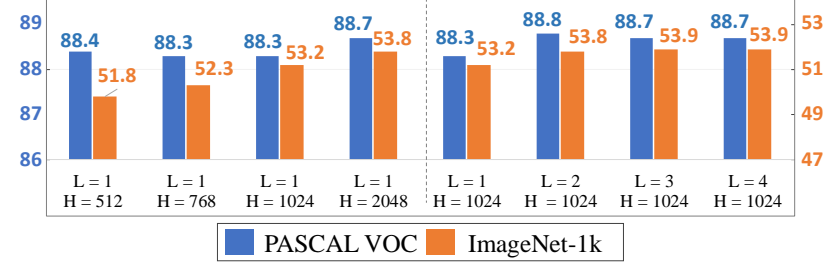

Figure 5: Ablations. (a) Pretraining Tasks: Bicaptioning improves over weaker pretraining tasks - forward captioning, token classification and masked language modeling. (b) Visual Backbone: Bigger visual backbones improve downstream performance - both, wider (R-50 w $2 \times$ ) and deeper (R-101). (c) Transformer Size: Larger transformers (wider and deeper) improve downstream performance.

supervised models and VirTex is unfair on both ends, as the former observes downstream images during pretraining, while the latter uses annotated images.

\subsection{Ablations}

The preceeding linear classification experiments demonstrate the effectiveness and data-efficiency of VirTex. In this section, we conduct ablation studies to isolate the effects of our pretraining setup and modeling decisions, and uncover performance trends to seed intuition for future work. We evaluate all ablations on PASCAL VOC and ImageNet-1k linear classification, as described in Section 4.1.

Pretraining Task Ablations: We choose bicaptioning task as it gives a dense supervisory signal per caption. To justify this choice, we form three pretraining tasks with sparser supervisory signal and compare them with bicaptioning:

- Forward Captioning: We remove the backward transformer decoder and only perform left-to-right captioning.

- Token Classification: We replace the textual head with a linear layer and perform multi-label classification $(\mathrm{Ta}-$ ble 1, row 2). We use the set of caption tokens as targets, completely ignoring the linguistic structure of captions.

- Masked Language Modeling (MLM): We use a single bidirectional transformer in the textual head, and perform BERT-like masked language modeling. We randomly mask $15 \%$ of input tokens, and train the model to predict ground-truth tokens of masked positions.

All textual heads with transformers have $L=1, H=2048$.

Results are shown in Figure 5(a). Bicaptioning outperforms forward captioning, indicating that denser supervi- 


\begin{tabular}{|c|c|c|c|c|c|c|c|c|c|c|c|c|c|c|}
\hline \multirow[b]{2}{*}{ Method } & \multirow{2}{*}{$\begin{array}{c}\text { Pretrain } \\
\text { Images }\end{array}$} & \multicolumn{6}{|c|}{ COCO Instance Segmentation } & \multicolumn{3}{|c|}{ LVIS Instance Segmentation } & \multicolumn{3}{|c|}{ PASCAL VOC Detection } & \multirow{2}{*}{$\frac{\text { iNat } 18}{\text { Top-1 }}$} \\
\hline & & $\mathrm{AP}_{\mathrm{all}}^{\mathrm{bbox}}$ & $\mathrm{AP}_{50}^{\mathrm{bbbx}}$ & $\mathrm{AP}_{75}^{\mathrm{bbox}}$ & $\mathrm{AP}_{\text {all }}^{\text {mask }}$ & $\mathrm{AP}_{50}^{\text {mask }}$ & $\mathrm{AP}_{75}^{\text {mask }}$ & $\mathrm{AP}_{\text {all }}^{\text {mask }}$ & $\mathrm{AP}_{50}^{\text {mask }}$ & $\mathrm{AP}_{75}^{\text {mask }}$ & $\mathrm{AP}_{\text {all }}^{\mathrm{bbox}}$ & $\mathrm{AP}_{50}^{\mathrm{bbox}}$ & $\mathrm{AP}_{75}^{\text {bbox }}$ & \\
\hline 1) Random Init & & 36.7 & 56.7 & 40.0 & 33.7 & 53.8 & 35.9 & 17.4 & 27.8 & 18.4 & 33.8 & 60.2 & 33.1 & 61.4 \\
\hline 2) IN-sup & $1.28 \mathrm{M}$ & 41.1 & 62.0 & 44.9 & 37.2 & 59.1 & 40.0 & 22.6 & 35.1 & 23.7 & 54.3 & 81.6 & 59.7 & 65.2 \\
\hline 3) IN-sup- $50 \%$ & $640 \mathrm{~K}$ & $40.3_{-0.8}$ & $61.0_{-1.0}$ & $44.0_{-0.9}$ & $36.6_{-0.6}$ & $58.0_{-1.1}$ & $39.3_{-0.7}$ & $21.2_{-1.4}$ & $33.3_{-1.8}$ & $22.3_{-1.4}$ & $52.1_{-2.2}$ & $80.4_{-1.2}$ & $57.0_{-2.7}$ & $63.2_{-2.0}$ \\
\hline 4) IN-sup- $10 \%$ & $128 \mathrm{~K}$ & $37.9_{-3.2}$ & $58.2_{-3.8}$ & $41.1_{-3.8}$ & $34.7_{-2.5}$ & $55.2_{-3.9}$ & $37.1_{-2.9}$ & $17.5_{-5.1}$ & $28.0_{-7.1}$ & $18.4_{-5.3}$ & $42.6_{-11.7}$ & $72.0_{-9.6}$ & $43.8_{-15.9}$ & $60.2_{-4.7}$ \\
\hline 5) MoCo-IN & $1.28 \mathrm{M}$ & $40.8_{-0.3}$ & $61.6_{-0.4}$ & $44.7_{-0.2}$ & $36.9-0.3$ & $58.4_{-0.7}$ & $39.7_{-0.3}$ & $22.8+0.2$ & $35.4_{+0.3}$ & $24.2+0.5$ & $56.1_{+1.8}$ & $81.5_{-0.1}$ & $62.4_{+0.7}$ & $63.2_{-1.7}$ \\
\hline 6) $\mathrm{MoCo}-\mathrm{COCO}$ & $118 \mathrm{~K}$ & $38.5_{-0.6}$ & $58.5_{-3.5}$ & $42.0_{-2.9}$ & $35.0_{-2.2}$ & $55.6_{-3.5}$ & $37.5_{-2.5}$ & $20.7_{-1.9}$ & $32.3_{-2.8}$ & $21.9_{-1.8}$ & $47.6_{-6.7}$ & $75.4_{-6.2}$ & $51.0_{-8.7}$ & $60.5_{-4.4}$ \\
\hline 7) VirTex & $118 \mathrm{~K}$ & $40.9_{-0.2}$ & $61.7_{-0.3}$ & $44.8_{-0.1}$ & $36.9-0.3$ & $58.4_{-0.7}$ & $39.7_{-0.3}$ & $23.0_{+0.4}$ & $35.4_{+0.4}$ & $24.3_{+0.6}$ & $55.3_{+1.0}$ & $81.3_{-0.3}$ & $61.0_{+1.3}$ & $63.4_{-1.4}$ \\
\hline
\end{tabular}

Table 3: Fine-tuning Tasks for Transfer: We compare VirTex with different pretraining methods across four downstream tasks. For each task, all methods use the same architecture. We initialize the ResNet-50 backbone weights from pretraining (except Random Init), which are then fine-tuned end-to-end. Performance gaps with IN-sup are shown on the side. On all tasks, VirTex significantly outperforms all methods that use similar amount of pretraining images. VirTex closely matches or exceeds ImageNet supervised and self-supervised methods, despite using $10 \times$ fewer pretraining images.

sory signal from bidirectional modeling is beneficial. Bicaptioning and forward captioning both outperform token classification, demonstrating that learning to model the sequential structure of language improves visual features.

MLM performs quite worse than all three methods, possibly due to poor sample efficiency (discussed in Section 3) It may benefit from longer training schedules, however we leave this for future work due to computational constraints. Visual Backbone Ablations: Bigger visual backbones tend to show improvements on many vision tasks [2, 9, 103]. We investigate whether VirTex models with bigger visual backbones can improve downstream performance. We train three VirTex models with $L=1, H=1024$ textual heads, and different visual backbones: (a) ResNet-50 (default), (b) ResNet-50 w $2 \times$ [104] ( $2 \times$ channel width), and (c) ResNet$101(2 \times$ depth). We observe that bigger visual backbones better results on VOC07, however the trends are opposite on ImageNet (Figure 5(b)). We believe it to be an optimization issue. See Appendix A.2 for comparison on other tasks.

Transformer Size Ablations: Prior work in language modeling has shown that larger Transformers tend to learn better textual features [80-83]. We investigate whether this holds for VirTex: do larger transformers in the textual head cause the visual backbone to learn better visual features? As discussed in Section 3, we may scale our textual head by increasing its width (hidden size $H$ ) or its depth (number of layers $L$ ). We investigate both, training VirTex models with: - Fixed $L=1$, increasing $H \in\{512,768,1024,2048\}$.

- Fixed $H=1024$, increasing $L \in\{1,2,3,4\}$.

Results are shown in Figure 5(c) - increasing transformer size, both width and depth, generally improves downstream performance. Performance degrades slightly with very deep transformers $(L=4)$, indicating overfitting. We hope that massive transformers with billions of parameters will help when scaling VirTex to large-scale, more noisy image-text paired datasets [37-39] that are larger than COCO Captions.

\subsection{Fine-tuning Tasks for Transfer}

So far we have evaluated VirTex using features extracted from frozen visual backbones. Another common mechanisms for transfer learning is fine-tuning, where the entire visual backbone is updated for the downstream task.

We evaluate features learned using VirTex on four downstream tasks with fine-tuning: (a) Instance Segmentation on COCO [30]; (b) Instance Segmentation on LVIS [31]; and (c) Object Detection on PASCAL VOC [99]; (d) Finegrained Classification on iNaturalist 2018 [107]. In all these experiments, we use the VirTex model with ResNet-50 visual backbone and a textual head with $L=1, H=2048$.

Baselines: Our main baselines are ImageNet-supervised (IN-sup) and MoCo. We consider three variants of IN-sup pretrained with $\{10,50,100\} \%$ of ImageNet images (Figure 4). Similarly for MoCo, we consider both MoCo-IN (Table 2) and MoCo-COCO (Table 1). We also include Random Init baseline, trained from scratch on downstream task.

We follow the same evaluation protocol as MoCo [24] for all four tasks. We use Detectron2 [101] for tasks $(a, b, c)$. Our IN-sup- $100 \%$ results are slightly better than those reported in [24] - we use pretrained ResNet-50 model from torchvision, whereas they used the MSRA ResNet-50 model from Detectron [108]. We briefly describe implementation details that differ from default Detectron2 settings. Refer Appendix A.3 for full details.

COCO Instance Segmentation: We train Mask RCNN [9] models with ResNet-50-FPN backbones [109]. We initialize backbone with pretrained weights, train on train2017 split, and evaluate on val2017 split. We finetune all layers end-to-end with BN layers synchronized across GPUs [110] (SyncBN). We also use SyncBN in FPN layers. We train with batch size 16 distributed across 8 GPUs, following $2 \times$ schedule ( $180 \mathrm{~K}$ iterations with initial LR 0.02 , multiplied by 0.1 at iterations $120 \mathrm{~K}$ and $160 \mathrm{~K}$ ). 


\begin{tabular}{lcccc}
\hline Backbone & Depth & Width & CIDEr & SPICE \\
\hline R-50 & 1 & 512 & 103.2 & 19.3 \\
R-50 & 1 & 768 & 103.7 & 19.6 \\
R-50 & 1 & 1024 & 103.5 & 19.8 \\
R-50 & 1 & 2048 & $\mathbf{1 0 4 . 2}$ & $\mathbf{1 9 . 9}$ \\
\hline R-50 & 1 & 1024 & 103.5 & 19.8 \\
R-50 & 2 & 1024 & $\mathbf{1 0 6 . 9}$ & $\mathbf{2 0 . 0}$ \\
R-50 & 3 & 1024 & 104.3 & 19.5 \\
R-50 & 4 & 1024 & 103.8 & 19.2 \\
\hline R-50 w2 $\times$ & 1 & 1024 & 102.7 & 19.6 \\
R-101 & 1 & 1024 & $\mathbf{1 0 6 . 6}$ & $\mathbf{2 0 . 1}$ \\
\hline
\end{tabular}
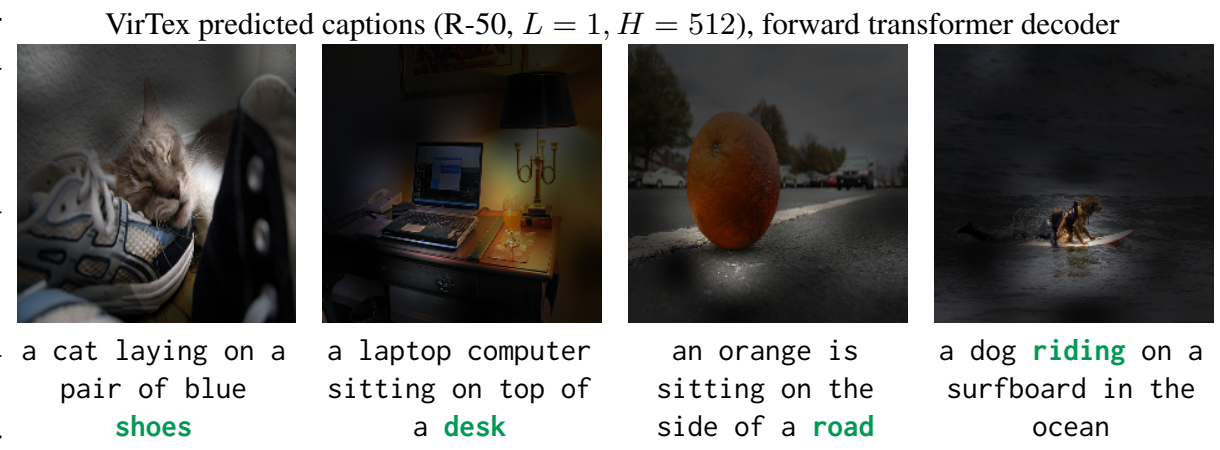

Figure 6: Image Captioning: We report image captioning performance (CIDEr [105] and SPICE [106]) of VirTex models on COCO val2017 split - all variants show modest performance. We also show some predicted captions on the right. For the highlighted word, we visualize decoder attention weights from the textual head on the input image. Our model focuses on relevant image regions to predict objects (shoes, desk), background (road) as well as actions (riding).

LVIS Instance Segmentation: The LVIS dataset provides instance segmentation labels for a long tail of 1203 entrylevel object categories, and stresses the ability to recognize many object types from few training samples. We train Mask R-CNN models with ResNet-50-FPN backbones on train_v1. $\theta$ and evaluate on val_v1. 0 split. Following MoCo settings, we keep BN parameters frozen for all INsup baselines. We train with $2 \times$ schedule as $\mathrm{COCO}$, use class resampling and test-time hyperparameters $(0.0$ score threshold and 300 detections per image) same as [31].

PASCAL VOC Detection: We train Faster R-CNN [111] models with ResNet-50-C4 backbones on trainval07+12 split, and evaluate on test2007 split. Like COCO, we finetune all models with SyncBN. We train for $24 \mathrm{~K}$ iterations, including linear LR warmup for first 100 iterations. We set the maximum LR as 0.02 , that is divided by 10 at iterations $18 \mathrm{~K}$ and $22 \mathrm{~K}$. We distribute training across 8 GPUs, with batch size 2 per GPU. We use gradient checkpointing [112, 113 ] to reduce the heavy memory footprint of these models and train them with desired batch size on our 12 GB GPUs.

iNaturalist 2018 Fine-grained Classification: The iNaturalist 2018 dataset provides labeled images for 8142 finegrained categories, with a long-tailed distribution. We finetune the pretrained ResNet-50 with a linear layer end-toend. We train on train2018 split and evaluate on val2018 split, following training setup from torchvision - we train for 100 epochs using SGD with momentum 0.9 and weight decay $10^{-4}$, and batch size 256 distributed across 8 GPUs. Fine-tuning uses LR 0.025 (and Random Init uses 0.1), which is multiplied by 0.1 at epochs 70 and 90 .

Results: We show results in Table 3. VirTex matches or exceeds ImageNet-supervised pretraining and MoCo-IN on all tasks (row 2,5 vs. 7) despite using $10 \times$ fewer pretraining images. Moreover, VirTex significantly outperforms methods that use similar, or more pretraining images (row 3, 4,6 vs. 7), indicating its superior data-efficiency. Among all tasks, VirTex shows significant improvements on LVIS, that shows the effectiveness of natural language annotations in capturing the long tail of visual concepts in the real world.

\subsection{Image Captioning}

Our goal is to learn transferable visual features via textual supervision. To do so, we use image captioning as a pretraining task. Although our goal is not to advance the state-of-the-art in image captioning, in Figure 6 we show quantitative and qualitative results of VirTex models trained from scratch on COCO. All models show modest performance, far from current state-of-the-art methods, that commonly involve some pretraining. However, captioning metrics are known to correlate weakly with human judgement - we surpass human performance on COCO.

We show some predicted captions by VirTex (R-50, $L=$ $1, H=512)$ model. We apply beam search on the forward transformer decoder ( 5 beams) to decode most likely captions. The decoder attention module in this transformer attends over a $7 \times 7$ grid of image features through $A=8$ heads at each time-step for predicting a token. We average these $7 \times 7$ attention weights over all the heads, and overlay them on $224 \times 224$ input image (via bicubic upsampling).

In Figure 6, we show visualizations for some tokens. We observe that our model attends to relevant image regions for making predictions, indicating that VirTex learns meaningful visual features with good semantic understanding.

\section{Conclusion}

We have shown that learning visual representations using textual annotations can be competitive to methods based on supervised classification and self-supervised learning on ImageNet. We solely focus on downstream vision tasks future works can explore other tasks that transfer both the visual backbone and the textual head. Finally, the usage of captions opens a clear pathway to scaling our approach to web-scale image-text pairs, that are orders of magnitude larger, albeit more noisy than COCO Captions. 


\section{Acknowledgments}

We thank Harsh Agrawal, Mohamed El Banani, Richard Higgins, Nilesh Kulkarni and Chris Rockwell for helpful discussions and feedback on the paper. We thank Ishan Misra for discussions regarding PIRL/SwAV evaluation protocol; Saining Xie for discussions about replicating iNaturalist evaluation as MoCo; Ross Girshick and Yuxin Wu for help with Detectron2 model zoo; Georgia Gkioxari for suggesting the Instance Segmentation pretraining task ablation; and Stefan Lee for suggestions on figure aesthetics. We thank Jia Deng for access to extra GPUs during project development; and UMich ARC-TS team for support with GPU cluster management. Finally, we thank all the Starbucks outlets in Ann Arbor for many hours of free WiFi. This work was partially supported by the Toyota Research Institute (TRI). However, note that this article solely reflects the opinions and conclusions of its authors and not TRI or any other Toyota entity.

\section{References}

[1] Alex Krizhevsky, Ilya Sutskever, and Geoffrey E Hinton, "Imagenet classification with deep convolutional neural networks," in NeurIPS, 2012. 1

[2] Kaiming He, Xiangyu Zhang, Shaoqing Ren, and Jian Sun, "Deep residual learning for image recognition," in $C V P R$, 2016. 1, 3, 7

[3] Jia Deng, Wei Dong, Richard Socher, Li-Jia Li, Kai Li, and Li Fei-Fei, "Imagenet: A large-scale hierarchical image database," in CVPR, 2009. 1

[4] Olga Russakovsky, Jia Deng, Hao Su, Jonathan Krause, Sanjeev Satheesh, Sean Ma, Zhiheng Huang, Andrej Karpathy, Aditya Khosla, Michael Bernstein, et al., "Imagenet large scale visual recognition challenge," IJCV, 2015. 1,5

[5] Jeff Donahue, Yangqing Jia, Oriol Vinyals, Judy Hoffman, Ning Zhang, Eric Tzeng, and Trevor Darrell, "Decaf: A deep convolutional activation feature for generic visual recognition," in ICML, 2014. 1

[6] Ali Sharif Razavian, Hossein Azizpour, Josephine Sullivan, and Stefan Carlsson, "Cnn features off-the-shelf: an astounding baseline for recognition," in CVPR Workshops, 2014. 1

[7] Ross Girshick, Jeff Donahue, Trevor Darrell, and Jitendra Malik, "Rich feature hierarchies for accurate object detection and semantic segmentation," in CVPR, 2014. 1

[8] Jonathan Long, Evan Shelhamer, and Trevor Darrell, "Fully convolutional networks for semantic segmentation," in CVPR, 2015. 1

[9] Kaiming He, Georgia Gkioxari, Piotr Dollár, and Ross Girshick, "Mask R-CNN," in ICCV, 2017. 1, 7

[10] Oriol Vinyals, Alexander Toshev, Samy Bengio, and Dumitru Erhan, "Show and tell: A neural image caption generator," in CVPR, 2015. 1, 3, 4

[11] Andrej Karpathy and Li Fei-Fei, "Deep visual-semantic alignments for generating image descriptions," in CVPR, 2015. 4
[12] Jeffrey Donahue, Lisa Anne Hendricks, Sergio Guadarrama, Marcus Rohrbach, Subhashini Venugopalan, Kate Saenko, and Trevor Darrell, "Long-term recurrent convolutional networks for visual recognition and description," in $C V P R, 2015$. 1, 3

[13] Stanislaw Antol, Aishwarya Agrawal, Jiasen Lu, Margaret Mitchell, Dhruv Batra, C Lawrence Zitnick, and Devi Parikh, "VQA: Visual question answering," in ICCV, 2015. 1,2

[14] Yuke Zhu, Oliver Groth, Michael Bernstein, and Li Fei-Fei, "Visual7w: Grounded question answering in images," in CVPR, 2016. 1, 2

[15] Carl Doersch, Abhinav Gupta, and Alexei A Efros, "Unsupervised visual representation learning by context prediction," in ICCV, 2015. 1, 2

[16] Deepak Pathak, Philipp Krahenbuhl, Jeff Donahue, Trevor Darrell, and Alexei A Efros, "Context encoders: Feature learning by inpainting," in $C V P R, 2016.2$

[17] Richard Zhang, Phillip Isola, and Alexei A Efros, "Colorful image colorization," in $E C C V, 2016.2$

[18] Richard Zhang, Phillip Isola, and Alexei A Efros, "Splitbrain autoencoders: Unsupervised learning by crosschannel prediction," in CVPR, 2017. 2

[19] Spyros Gidaris, Praveer Singh, and Nikos Komodakis, "Unsupervised representation learning by predicting image rotations," in ICLR, 2018. 2

[20] Aaron van den Oord, Yazhe Li, and Oriol Vinyals, "Representation learning with contrastive predictive coding," arXiv preprint arXiv: $1807.03748,2018.2$

[21] Yonglong Tian, Dilip Krishnan, and Phillip Isola, "Contrastive multiview coding," in ECCV, 2020. 1, 2

[22] Priya Goyal, Dhruv Mahajan, Abhinav Gupta, and Ishan Misra, "Scaling and benchmarking self-supervised visual representation learning," in $C V P R, 2019.1,4,5,13$

[23] Olivier J Hénaff, Ali Razavi, Carl Doersch, SM Eslami, and Aaron van den Oord, "Data-efficient image recognition with contrastive predictive coding," arXiv preprint arXiv:1905.09272, 2019. 2

[24] Kaiming He, Haoqi Fan, Yuxin Wu, Saining Xie, and Ross Girshick, "Momentum contrast for unsupervised visual representation learning," in $C V P R, 2020.1,2,5,6,7,14$

[25] Ishan Misra and Laurens van der Maaten, "Self-supervised learning of pretext-invariant representations," in CVPR, 2020. $1,5,13$

[26] Ting Chen, Simon Kornblith, Mohammad Norouzi, and Geoffrey Hinton, "A simple framework for contrastive learning of visual representations," in ICML, 2020. 1, 2

[27] Mathilde Caron, Piotr Bojanowski, Armand Joulin, and Matthijs Douze, "Deep clustering for unsupervised learning of visual features," in ECCV, 2018. 1, 2

[28] Mathilde Caron, Piotr Bojanowski, Julien Mairal, and Armand Joulin, "Unsupervised pre-training of image features on non-curated data," in ICCV, 2019. 1

[29] Ashish Vaswani, Noam Shazeer, Niki Parmar, Jakob Uszkoreit, Llion Jones, Aidan N Gomez, Łukasz Kaiser, and Illia Polosukhin, "Attention is all you need," in NeurIPS, 2017. 1, 2, 3

[30] Tsung-Yi Lin, Michael Maire, Serge Belongie, James Hays, Pietro Perona, Deva Ramanan, Piotr Dollár, and C Lawrence Zitnick, "Microsoft COCO: Common objects 
in context," in $E C C V$, 2014. 1, 5, 7, 13

[31] Agrim Gupta, Piotr Dollar, and Ross Girshick, "LVIS: A dataset for large vocabulary instance segmentation," in CVPR, 2019. 1, 7, 8

[32] Jia Deng, Olga Russakovsky, Jonathan Krause, Michael S Bernstein, Alex Berg, and Li Fei-Fei, "Scalable multi-label annotation," in SIGCHI, 2014. 1

[33] Ranjay A Krishna, Kenji Hata, Stephanie Chen, Joshua Kravitz, David A Shamma, Li Fei-Fei, and Michael S Bernstein, "Embracing error to enable rapid crowdsourcing," in CHI, 2016. 1

[34] Micah Hodosh, Peter Young, and Julia Hockenmaier, "Framing image description as a ranking task: Data, models and evaluation metrics," JAIR, 2013. 2

[35] Peter Young, Alice Lai, Micah Hodosh, and Julia Hockenmaier, "From image descriptions to visual denotations: New similarity metrics for semantic inference over event descriptions," TACL, 2014. 2

[36] Xinlei Chen, Hao Fang, Tsung-Yi Lin, Ramakrishna Vedantam, Saurabh Gupta, Piotr Dollár, and C Lawrence Zitnick, "Microsoft COCO captions: Data collection and evaluation server," arXiv preprint arXiv:1504.00325, 2015. $2,4,5,13$

[37] Piyush Sharma, Nan Ding, Sebastian Goodman, and Radu Soricut, "Conceptual captions: A cleaned, hypernymed, image alt-text dataset for automatic image captioning," in ACL, 2018. 2, 7

[38] Junhua Mao, Jiajing Xu, Kevin Jing, and Alan L Yuille, "Training and evaluating multimodal word embeddings with large-scale web annotated images," in NIPS, 2016.

[39] Vicente Ordonez, Girish Kulkarni, and Tamara L. Berg, "Im2Text: Describing images using 1 million captioned photographs," in NIPS, 2011. 2, 7

[40] Ang Li, Allan Jabri, Armand Joulin, and Laurens van der Maaten, "Learning visual n-grams from web data," in ICCV, 2017. 2

[41] Bart Thomee, David A Shamma, Gerald Friedland, Benjamin Elizalde, Karl Ni, Douglas Poland, Damian Borth, and Li-Jia Li, "YFCC100M: The new data in multimedia research," Communications of the ACM, 2016. 2

[42] Chen Sun, Abhinav Shrivastava, Saurabh Singh, and Abhinav Gupta, "Revisiting unreasonable effectiveness of data in deep learning era," in $I C C V$, 2017. 2

[43] Alexander Kolesnikov, Lucas Beyer, Xiaohua Zhai, Joan Puigcerver, Jessica Yung, Sylvain Gelly, and Neil Houlsby, "Large scale learning of general visual representations for transfer," arXiv preprint arXiv:1912.11370, 2019.

[44] Qizhe Xie, Eduard Hovy, Minh-Thang Luong, and Quoc V Le, "Self-training with noisy student improves ImageNet classification," in CVPR, 2020. 2

[45] Dhruv Kumar Mahajan, Ross B. Girshick, Vignesh Ramanathan, Kaiming He, Manohar Paluri, Yixuan Li, Ashwin Bharambe, and Laurens van der Maaten, "Exploring the limits of weakly supervised pretraining," in ECCV, 2018. 2, 5

[46] I Zeki Yalniz, Hervé Jégou, Kan Chen, Manohar Paluri, and Dhruv Mahajan, "Billion-scale semi-supervised learning for image classification," arXiv preprint arXiv:1905.00546, 2019. 2
[47] Mehdi Noroozi and Paolo Favaro, "Unsupervised learning of visual representations by solving jigsaw puzzles," in ECCV, 2016. 2

[48] Jeff Donahue and Karen Simonyan, "Large scale adversarial representation learning," in NeurIPS, 2019. 2

[49] Michael Gutmann and Aapo Hyvärinen, "Noise-contrastive estimation: A new estimation principle for unnormalized statistical models," in AISTATS, 2010. 2

[50] Raia Hadsell, Sumit Chopra, and Yann LeCun, "Dimensionality reduction by learning an invariant mapping," in CVPR, 2006. 2

[51] Mang Ye, Xu Zhang, Pong C Yuen, and Shih-Fu Chang, "Unsupervised embedding learning via invariant and spreading instance feature," in CVPR, 2019. 2

[52] Zhirong Wu, Yuanjun Xiong, Stella Yu, and Dahua Lin, "Unsupervised feature learning via non-parametric instance-level discrimination," 2018. 2

[53] Philip Bachman, R Devon Hjelm, and William Buchwalter, "Learning representations by maximizing mutual information across views," in NeurIPS, 2019. 2

[54] R Devon Hjelm, Alex Fedorov, Samuel Lavoie-Marchildon, Karan Grewal, Phil Bachman, Adam Trischler, and Yoshua Bengio, "Learning deep representations by mutual information estimation and maximization," ICLR, 2019. 2

[55] Trieu H Trinh, Minh-Thang Luong, and Quoc V Le, "Selfie: Self-supervised pretraining for image embedding," arXiv preprint arXiv:1906.02940, 2019. 2

[56] Chengxu Zhuang, Alex Lin Zhai, and Daniel Yamins, "Local aggregation for unsupervised learning of visual embeddings," in ICCV, 2019. 2

[57] Junnan Li, Pan Zhou, Caiming Xiong, Richard Socher, and Steven C.H. Hoi, "Prototypical contrastive learning of unsupervised representations," arXiv preprint arXiv:2005.04966, 2020. 6

[58] Mathilde Caron, Ishan Misra, Julien Mairal, Priya Goyal, Piotr Bojanowski, and Armand Joulin, "Unsupervised learning of visual features by contrasting cluster assignments," in NeurIPS, 2020. 2, 5, 6, 13

[59] Yash Goyal, Tejas Khot, Douglas Summers-Stay, Dhruv Batra, and Devi Parikh, "Making the V in VQA matter: Elevating the role of image understanding in visual question answering," in CVPR, 2017. 2

[60] Drew A Hudson and Christopher D Manning, "GQA: A new dataset for real-world visual reasoning and compositional question answering," in CVPR, 2019. 2

[61] Alane Suhr, Stephanie Zhou, Ally Zhang, Iris Zhang, Huajun Bai, and Yoav Artzi, "A corpus for reasoning about natural language grounded in photographs," in ACL, 2019. 2

[62] Rowan Zellers, Yonatan Bisk, Ali Farhadi, and Yejin Choi, "From recognition to cognition: Visual commonsense reasoning," in CVPR, 2019. 2

[63] Sahar Kazemzadeh, Vicente Ordonez, Mark Matten, and Tamara Berg, "Referitgame: Referring to objects in photographs of natural scenes," in EMNLP, 2014. 2

[64] Jacob Devlin, Ming-Wei Chang, Kenton Lee, and Kristina Toutanova, "BERT: Pre-training of deep bidirectional transformers for language understanding," in $N A A C L, 2019$. 2, $3,4,6,14$

[65] Hao Tan and Mohit Bansal, "LXMERT: Learning crossmodality encoder representations from transformers," in 
EMNLP, 2019. 2, 6

[66] Jiasen Lu, Dhruv Batra, Devi Parikh, and Stefan Lee, "ViLBERT: Pretraining task-agnostic visiolinguistic representations for vision-and-language tasks," in NeurIPS, 2019.

[67] Liunian Harold Li, Mark Yatskar, Da Yin, Cho-Jui Hsieh, and Kai-Wei Chang, "VisualBERT: A simple and performant baseline for vision and language," arXiv preprint arXiv:1908.03557, 2019.

[68] Weijie Su, Xizhou Zhu, Yue Cao, Bin Li, Lewei Lu, Furu Wei, and Jifeng Dai, "VL-BERT: Pre-training of generic visual-linguistic representations," 2020.

[69] Gen Li, Nan Duan, Yuejian Fang, Daxin Jiang, and Ming Zhou, "Unicoder-VL: A universal encoder for vision and language by cross-modal pre-training," AAAI, 2020.

[70] Yen-Chun Chen, Linjie Li, Licheng Yu, Ahmed El Kholy, Faisal Ahmed, Zhe Gan, Yu Cheng, and Jingjing Liu, "Uniter: Learning universal image-text representations," arXiv preprint arXiv:1909.11740, 2019.

[71] Luowei Zhou, Hamid Palangi, Lei Zhang, Houdong Hu, Jason J Corso, and Jianfeng Gao, "Unified vision-language pre-training for image captioning and VQA," AAAI, 2020.

[72] Xiujun Li, Xi Yin, Chunyuan Li, Pengchuan Zhang, Xiaowei Hu, Lei Zhang, Lijuan Wang, Houdong Hu, Li Dong, Furu Wei, et al., "Oscar: Object-semantics aligned pretraining for vision-language tasks," in ECCV, 2020. 2, 6

[73] Ranjay Krishna, Yuke Zhu, Oliver Groth, Justin Johnson, Kenji Hata, Joshua Kravitz, Stephanie Chen, Yannis Kalantidis, Li-Jia Li, David A Shamma, Michael S Bernstein, and Li Fei-Fei, "Visual genome: Connecting language and vision using crowdsourced dense image annotations," IJCV, 2017. 2, 6

[74] Peter Anderson, Xiaodong He, Chris Buehler, Damien Teney, Mark Johnson, Stephen Gould, and Lei Zhang, "Bottom-up and top-down attention for image captioning and visual question answering," in CVPR, 2018. 2, 6

[75] Mert Bulent Sariyildiz, Julien Perez, and Diane Larlus, "Learning visual representations with caption annotations," in $E C C V, 2020.3,6$

[76] Jonathan C Stroud, David A Ross, Chen Sun, Jia Deng, Rahul Sukthankar, and Cordelia Schmid, "Learning video representations from textual web supervision," in $E C C V$, 2020. 3

[77] Matthew E Peters, Mark Neumann, Mohit Iyyer, Matt Gardner, Christopher Clark, Kenton Lee, and Luke Zettlemoyer, "Deep contextualized word representations," in NAACL, 2018. 3, 4

[78] Zhilin Yang, Zihang Dai, Yiming Yang, Jaime Carbonell, Russ R Salakhutdinov, and Quoc V Le, "XLNet: Generalized autoregressive pretraining for language understanding," in NeurIPS, 2019. 3

[79] Alec Radford, Karthik Narasimhan, Tim Salimans, and Ilya Sutskever, "Improving language understanding by generative pre-training," 2018. 3, 4

[80] Alec Radford, Jeffrey Wu, Rewon Child, David Luan, Dario Amodei, and Ilya Sutskever, "Language models are unsupervised multitask learners," OpenAI Blog, 2019. 4, 7

[81] Tom B Brown, Benjamin Mann, Nick Ryder, Melanie Subbiah, Jared Kaplan, Prafulla Dhariwal, Arvind Neelakantan, Pranav Shyam, Girish Sastry, Amanda Askell, et al.,
"Language models are few-shot learners," arXiv preprint arXiv:2005.14165, 2020. 3

[82] Yinhan Liu, Myle Ott, Naman Goyal, Jingfei Du, Mandar Joshi, Danqi Chen, Omer Levy, Mike Lewis, Luke Zettlemoyer, and Veselin Stoyanov, "RoBERTa: A robustly optimized bert pretraining approach," arXiv preprint arXiv:1907.11692, 2019. 3

[83] Mohammad Shoeybi, Mostofa Patwary, Raul Puri, Patrick LeGresley, Jared Casper, and Bryan Catanzaro, "MegatronLM: Training multi-billion parameter language models using model parallelism," arXiv preprint arXiv:1909.08053, 2019. 3, 7

[84] Kevin Clark, Minh-Thang Luong, Quoc V. Le, and Christopher D. Manning, "ELECTRA: Pre-training text encoders as discriminators rather than generators," in ICLR, 2020. 3

[85] Dan Hendrycks and Kevin Gimpel, "Gaussian error linear units (gelus)," arXiv preprint arXiv:1606.08415, 2016. 3

[86] Jimmy Lei Ba, Jamie Ryan Kiros, and Geoffrey E Hinton, "Layer normalization," arXiv preprint arXiv:1607.06450, 2016. 4

[87] Nitish Srivastava, Geoffrey Hinton, Alex Krizhevsky, Ilya Sutskever, and Ruslan Salakhutdinov, "Dropout: a simple way to prevent neural networks from overfitting," JMLR, 2014. 4

[88] Hakan Inan, Khashayar Khosravi, and Richard Socher, "Tying word vectors and word classifiers: A loss framework for language modeling," arXiv preprint arXiv:1611.01462, 2016. 4

[89] Ofir Press and Lior Wolf, "Using the output embedding to improve language models," in EACL, 2017. 4

[90] Taku Kudo and John Richardson, "SentencePiece: A simple and language independent subword tokenizer and detokenizer for neural text processing," in EMNLP: System Demonstrations, 2018. 4

[91] Rico Sennrich, Barry Haddow, and Alexandra Birch, "Neural machine translation of rare words with subword units," in $A C L, 2016.4$

[92] Boris T Polyak, "Some methods of speeding up the convergence of iteration methods," USSR Computational Mathematics and Mathematical Physics, 1964. 4

[93] Ilya Sutskever, James Martens, George Dahl, and Geoffrey Hinton, "On the importance of initialization and momentum in deep learning," in ICML, 2013. 4

[94] Michael Zhang, James Lucas, Jimmy Ba, and Geoffrey E Hinton, "Lookahead optimizer: $\mathrm{k}$ steps forward, 1 step back," in NeurIPS, 2019. 4

[95] Sergey Ioffe and Christian Szegedy, "Batch normalization: Accelerating deep network training by reducing internal covariate shift," in ICML, 2015. 4

[96] Ilya Loshchilov and Frank Hutter, "SGDR: Stochastic gradient descent with warm restarts," arXiv preprint arXiv:1608.03983, 2016. 4

[97] Adam Paszke, Sam Gross, Francisco Massa, Adam Lerer, James Bradbury, Gregory Chanan, Trevor Killeen, Zeming Lin, Natalia Gimelshein, Luca Antiga, Alban Desmaison, Andreas Kopf, Edward Yang, Zachary DeVito, Martin Raison, Alykhan Tejani, Sasank Chilamkurthy, Benoit Steiner, Lu Fang, Junjie Bai, and Soumith Chintala, "Pytorch: An imperative style, high-performance deep learning library," 
in NeurIPS, 2019. 4

[98] Paulius Micikevicius, Sharan Narang, Jonah Alben, Gregory Diamos, Erich Elsen, David Garcia, Boris Ginsburg, Michael Houston, Oleksii Kuchaiev, Ganesh Venkatesh, et al., "Mixed precision training," in ICLR, 2018. 4

[99] Mark Everingham, Luc Van Gool, Christopher K. I. Williams, John M. Winn, and Andrew Zisserman, "The pascal visual object classes (VOC) challenge," IJCV, 2009. 4, 5,7

[100] Harsh Agrawal, Karan Desai, Yufei Wang, Xinlei Chen, Rishabh Jain, Mark Johnson, Dhruv Batra, Devi Parikh, Stefan Lee, and Peter Anderson, "nocaps: novel object captioning at scale," in Proceedings of the IEEE International Conference on Computer Vision, pp. 8948-8957, 2019. 5, 13

[101] Yuxin Wu, Alexander Kirillov, Francisco Massa, Wan-Yen Lo, and Ross Girshick, "Detectron2." https://github. com/facebookresearch/detectron2, 2019. 5, 7, 14

[102] Kaiming He, Ross Girshick, and Piotr Dollár, "Rethinking ImageNet pre-training," in ICCV, 2019. 5

[103] Saining Xie, Ross Girshick, Piotr Dollár, Zhuowen Tu, and Kaiming He, "Aggregated residual transformations for deep neural networks," in CVPR, 2017. 7

[104] Sergey Zagoruyko and Nikos Komodakis, "Wide residual networks," arXiv preprint arXiv:1605.07146, 2016. 7

[105] Ramakrishna Vedantam, C Lawrence Zitnick, and Devi Parikh, "CIDEr: Consensus-based image description evaluation," in CVPR, 2015. 8, 15

[106] Peter Anderson, Basura Fernando, Mark Johnson, and Stephen Gould, "SPICE: Semantic propositional image caption evaluation," in ECCV, 2016. 8

[107] Grant Van Horn, Oisin Mac Aodha, Yang Song, Yin Cui, Chen Sun, Alex Shepard, Hartwig Adam, Pietro Perona, and Serge Belongie, "The inaturalist species classification and detection dataset," in CVPR, 2018. 7

[108] Ross Girshick, Ilija Radosavovic, Georgia Gkioxari, Piotr Dollár, and Kaiming He, "Detectron." https ://gi thub. com/facebookresearch/detectron, 2018. 7

[109] Tsung-Yi Lin, Piotr Dollár, Ross Girshick, Kaiming He, Bharath Hariharan, and Serge Belongie, "Feature pyramid networks for object detection," in CVPR, 2017. 7

[110] Chao Peng, Tete Xiao, Zeming Li, Yuning Jiang, Xiangyu Zhang, Kai Jia, Gang Yu, and Jian Sun, "MegDet: A large mini-batch object detector," in CVPR, 2018. 7

[111] Shaoqing Ren, Kaiming He, Ross Girshick, and Jian Sun, "Faster R-CNN: Towards real-time object detection with region proposal networks," in NeurIPS, 2015. 8

[112] James Martens and Ilya Sutskever, "Training deep and recurrent networks with hessian-free optimization," in Neural networks: Tricks of the trade, 2012. 8

[113] Tianqi Chen, Bing Xu, Chiyuan Zhang, and Carlos Guestrin, "Training deep nets with sublinear memory cost," arXiv preprint arXiv:1604.06174, 2016. 8

[114] F. Pedregosa, G. Varoquaux, A. Gramfort, V. Michel, B. Thirion, O. Grisel, M. Blondel, P. Prettenhofer, R. Weiss, V. Dubourg, J. Vanderplas, A. Passos, D. Cournapeau, M. Brucher, M. Perrot, and E. Duchesnay, "Scikit-learn: Machine learning in Python," Journal of Machine Learning Research, vol. 12, pp. 2825-2830, 2011. 13

[115] Rong-En Fan, Kai-Wei Chang, Cho-Jui Hsieh, Xiang-Rui
Wang, and Chih-Jen Lin, "LIBLINEAR: A library for large linear classification," Journal of machine learning research, 2008. 13 


\section{Appendix A. Additional Experiments}

In this section, we describe additional implementation details about our experiments in Section 4. Our evaluation protocol is consistent with prior works on pretraining visual representations - we report differences where applicable.

\section{A.1. Image Classification with Linear Models}

PASCAL VOC: We use standard data augmentation on images from both trainval and test split - we resize the shorter edge to 256 pixels, and take a $224 \times 224$ center crop. We normalize images by ImageNet color (RGB mean $=[0.485,0.456,0.406], \mathrm{std}=[0.229,0.224,0.225])$.

Prior works $[22,25,58]$ train per-class SVMs for $C \in$ $\left[2^{-19}, 2^{-4}\right] \cup\left[10^{-7}, 10^{-2}\right]$ (26 values), and choose best SVM based on 3-fold cross-validation. In our initial evaluations, we observed that the best performing SVMs are typically trained with cost values $C \in\{0.01,0.1,1.0,10.0\}$. Based on this observation, we only use these values for faster evaluation. For training SVMs, we use scikitlearn [114] with LIBLINEAR [115] backend, default parameters are: LinearSVC (penalty=' 12 ', dual=True, max_iter $=2000$, tol $=1 e^{-4}$, class_weight $=\{1: 2,-1$ : $1\}$, loss='squared_hinge').

ImageNet-1k: For data augmentation during training, we randomly crop $20-100 \%$ of the original image size, with a random aspect ratio in $(4 / 3,3 / 4)$, resize to $224 \times 224$, apply random flip, and normalization by ImageNet color. During evaluation, we resize the shorter edge to 256 pixels and take a $224 \times 224$ center crop. We initialize the weights of the linear layer as $N(0.0,0.01)$, and bias values as 0 .

Note that we perform a small LR sweep separately for our VirTex model (ResNet-50 and $L=1, H=2048$ ), and ImageNet-supervised models. For Figure 4, best LR values for VirTex models is 0.3 (as mentioned in Section 4.1, and ImageNet-supervised models is 0.1 .

Annotation Cost Efficiency: Here, we provide details on our cost estimates for different methods in Table 1. For labels and masks, we use estimates reported by COCO [30], and for captions we use estimates reported by nocaps [100], collected in a similar fashion as COCO.

- Labels: We consider total time of Category Labeling and Instance Spotting steps in [30] ( $\sim 30 \mathrm{~K}$ hours). This estimate corresponds to $328 \mathrm{~K}$ images - we scale it for COCO Captions train2017 split (118K images).

- Masks: As reported in [30], it takes 22 worker hours for collecting 1000 instance segmentation masks. We use this estimate to compute time for $\sim 860 \mathrm{~K}$ masks in COCO train2017 split. The collection of masks is dependent on Category Labeling and Instance Spotting, we add the time for collecting labels in our total estimate.

- Captions: We use the median time per caption $(39.2 \mathrm{sec}-$ onds) as reported in [100] ( $\sim 151 \mathrm{~K}$ captions) to estimate the cost of collecting $(118 \mathrm{~K} \times 5)$ captions in COCO.

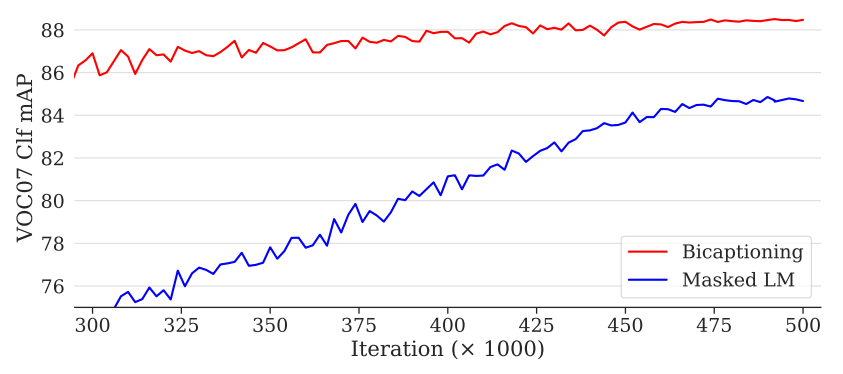

Figure 7: Bicaptioning vs. Masked Language Modeling: We compare VOC07 mAP of Bicaptioning and Masked LM pretraining tasks. We observe that Masked LM converges slower than Bicaptioning, indicating poor sample efficiency.

Data Efficiency: We train our ImageNet-supervised models on randomly sampled subsets of ImageNet (1\%, $2 \% 5 \%$, $10 \%, 20 \%, 50 \%)$. We sample training examples such that the class distribution remains close to $100 \%$ ImageNet. For VirTex models, we randomly sample $10 \%, 20 \%, 50 \%$, and $100 \%$ of COCO Captions [36] - we do not use any class labels to enforce uniform class distribution. Note that this may put ImageNet-supervised models at an advantage.

We train our ImageNet-supervised models by following the exact setup used to train the publicly available ResNet50 model in torchvision. We use SGD with momentum 0.9 and weight decay $10^{-4}$. We use a batch size of 256 , and perform distributed training across 8 GPUs (batch size 32 per GPU). We train for 90 epochs, with an initial learning rate 0.1 , that is divided by 10 at epochs 30 and 60 . We keep the number of training epochs fixed for models trained on smaller subsets of ImageNet (else they tend to overfit). For VirTex models, we scale training iterations according to the size of the sampled training set.

Comparison: ImageNet vs. Cropped COCO. Note that the ImageNet images mostly contain a single object (commonly called iconic images). On the other hand, $\mathrm{COCO}$ dataset contains $\sim 2.9$ object classes and $\sim 5.7$ instances per image. It may seem that VirTex requires fewer images than ImageNet-supervised models as they contain multiple objects per image. Here, we make an additional comparison to control the varying image statistics between datasets.

Specifically, we crop objects from COCO images and create a dataset of $860 \mathrm{~K}$ iconic images. We randomly expand bounding boxes on all edges by $0-30$ pixels before cropping, to mimic ImageNet-like images. We train a ResNet-50 with same hyperparameters as ImageNetsupervised models, described above. It achieves $\mathbf{7 9 . 1}$ VOC07 mAP (vs. 88.7 VirTex). This shows that the dataefficiency of VirTex does not entirely stem from using scene images with multiple objects. 


\begin{tabular}{llllll}
\hline \multirow{2}{*}{ Backbone } & VOC07 & IN-1k & & \multicolumn{3}{c}{ PASCAL VOC Detection } \\
\cline { 5 - 7 } & mAP & Top-1 & $\mathrm{AP}_{\text {all }}^{\text {bbox }}$ & $\mathrm{AP}_{50}^{\text {bbox }}$ & $\mathrm{AP}_{75}^{\text {bbox }}$ \\
\hline ResNet-50 & 88.3 & 53.2 & 55.2 & 81.2 & 60.8 \\
ResNet-50 w2 & $88.5_{+0.2}$ & $52.9_{-0.3}$ & $56.6_{+1.4}$ & $82.0_{+0.8}$ & $62.8_{+2.0}$ \\
ResNet-101 & $88.7_{+0.4}$ & $52.0_{-1.2}$ & $57.9_{+2.7}$ & $82.0_{+0.8}$ & $63.6_{+2.8}$ \\
\hline
\end{tabular}

Table 4: Additional Evaluations for Backbone Ablations. We compare VirTex models ( $L=1, H=1024)$ with different visual backbones. We observe that larger backbones generally improve downstream performance.

\section{A.2. Ablations}

Bicaptioning vs. Masked Language Modeling. In our pretraining task ablations (Section 4.2), we observed that Masked Language Modeling performs quite worse than all other pretraining tasks on downstream linear classification performance. This issue arises from the poor sample efficiency of Masked LM, discussed in Section 3.

For more evidence, we inspect VOC07 mAP of Masked LM, validated periodically during training. In Figure 7, we compare this with VOC07 mAP of Bicaptioning. Both models use $L=1, H=2048$ textual heads. We find that Masked LM indeed converges slower than bicaptioning, as it receives weaker supervision per training caption - only corresponding to masked tokens. We believe that a longer training schedule may lead to MLM outperforming bicaptioning, based on its success in language pretraining [64].

Additional Evaluation: Backbone Ablations. In our backbone ablations (Figure 5), we observed that larger visual backbones improve VOC07 classification performance. However, the performance trend for ImageNet-1k linear classification is opposite. We think this is an optimization issue - the hyperparameters chosen for ResNet-50 may not be optimal for other backbones. To verify our claims, we evaluate these models on PASCAL VOC object detection.

In Table 4, we observe that the performance trends of PASCAL VOC object detection match with VOC07 classification. Hence, we conclude that using larger visual backbones can improve downstream performance.

\section{A.3. Fine-tuning Tasks for Transfer}

We described the main details for downstream finetuning tasks in Section 4.3. We provide config files in Detectron2 [101] format to exactly replicate our downstream fine-tuning setup for COCO (Table 5), PASCAL VOC (Table 6), LVIS (Table 7). We apply modified hyperparameters on top of base config files available at:

github.com/facebookresearch/detectron2 a b267c6

iNaturalist 2018 Fine-grained Classification: We use data augmentation and weight initialization same as ImageNet$1 \mathrm{k}$ linear classification (Section A.1). Despite a long-tailed distribution like LVIS, we do not perform class balanced resampling, following the evaluation setup of MoCo [24].

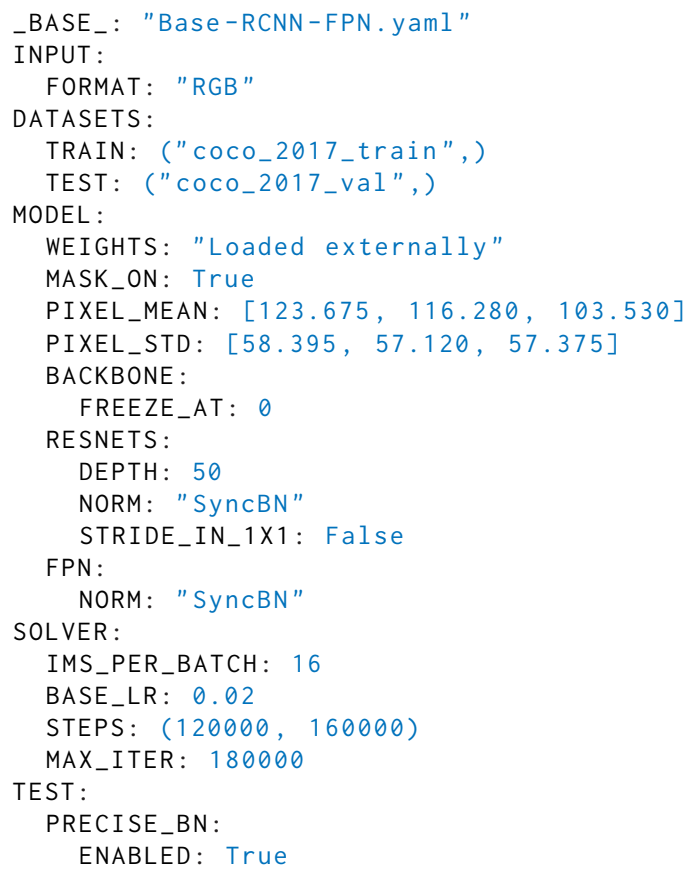

Table 5: COCO Instance Segmentation: Detectron2 config parameters that differ from base config file.

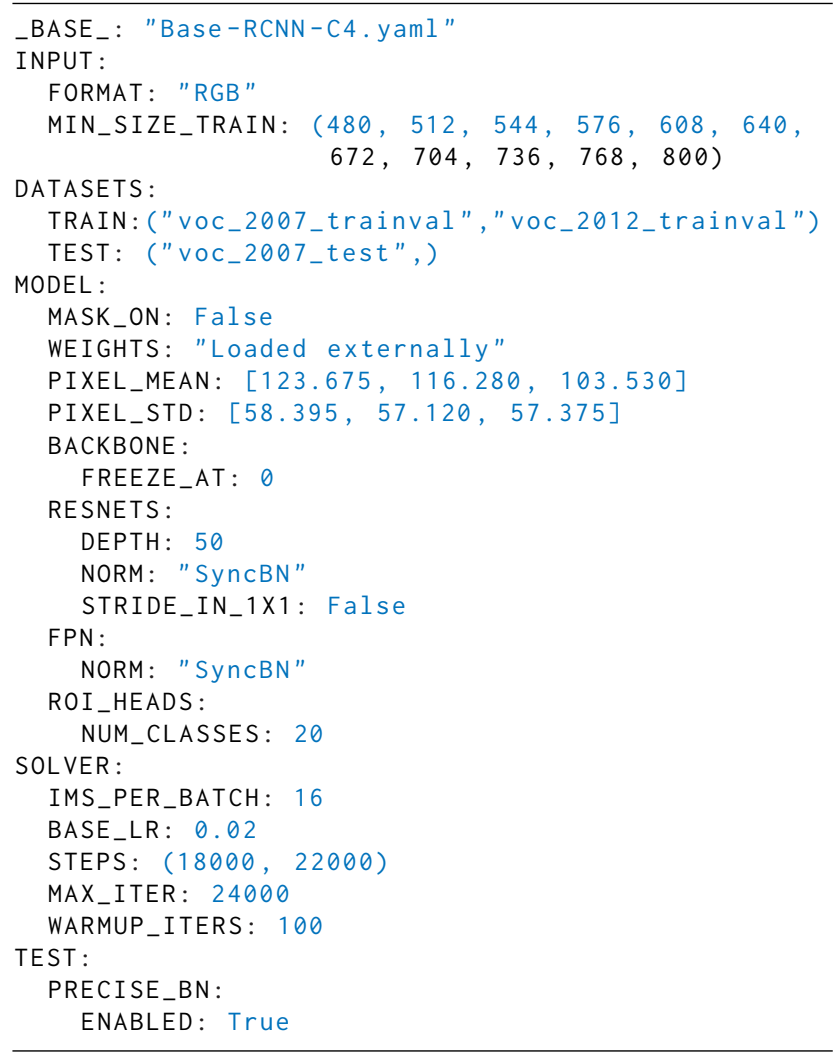

Table 6: PASCAL VOC Object Detection: Detectron2 config parameters that differ from base config file. 


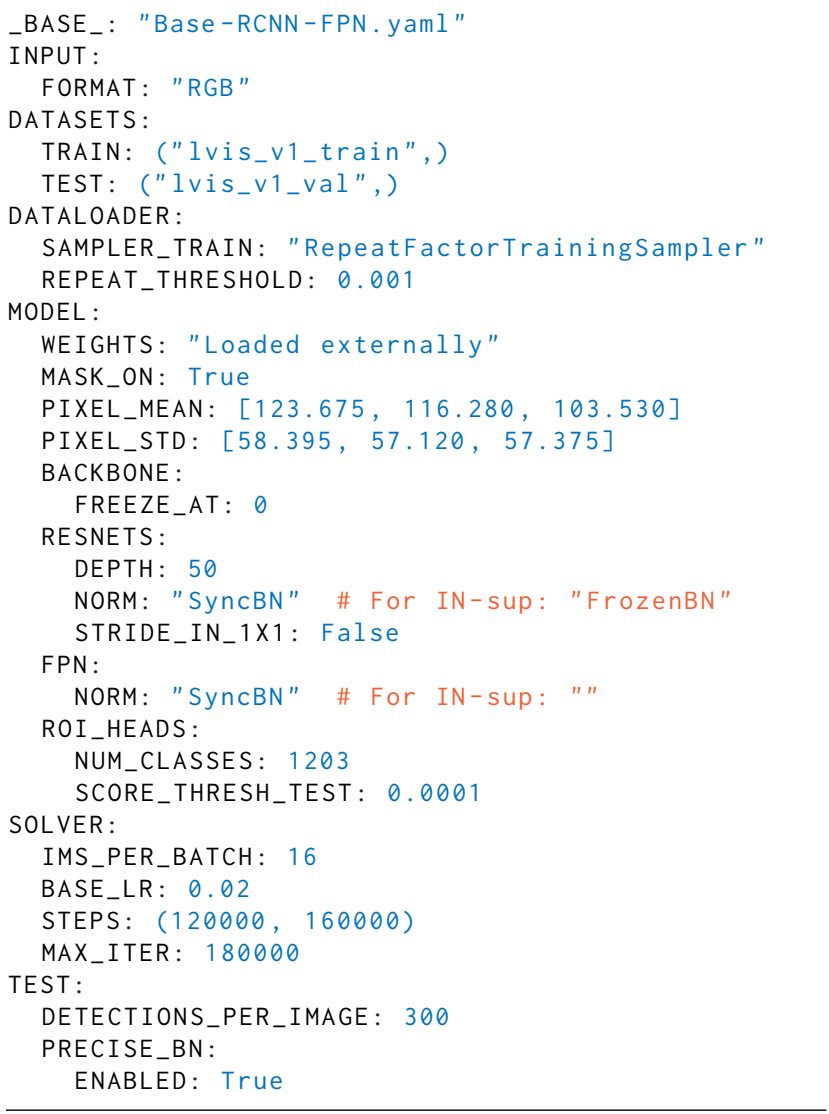

Table 7: LVIS Instance Segmentation: Detectron2 config parameters that differ from base config file.

LVIS v0.5 Instance Segmentation: In Section 4.3, we evaluated VirTex and baseline methods on LVIS Instance Segmentation task using LVIS v1. 0 train and val splits. One of our baselines, MoCo, conducted this evaluation using LVIS v 0.5 splits. For completeness, we report additional results on LVIS $\vee 0.5$ split. The main changes in config (Table 7) following original LVIS $v 0.5$ baselines are: NUM_CLASSES: 1230 and SCORE_THRESHOLD_TEST: 0.0

Results are shown in Table 8. We observe the VirTex significantly outperforms all baseline methods on LVIS v 0.5 split, similar to evaluation on LVIS v1. $\theta$ split.

\section{A.4. Selecting Best Checkpoint by VOC07 mAP}

As described in Section 3, we observed that image captioning performance has an imprecise correlation with performance on downstream vision tasks. Hence, we select our best checkpoint based on VOC07 classification mAP.

In Figure 8, we compare validation metrics of our best VirTex model (ResNet-50, $L=1, H=2048$ ). We observe the trends of VOC07 mAP and CIDEr [105] score of the forward transformer decoder. We observe that an improvement in captioning performance indicates an improve-

\begin{tabular}{lllll}
\hline \multirow{2}{*}{ Method } & \multirow{2}{*}{$\begin{array}{c}\text { Pretrain } \\
\text { Images }\end{array}$} & \multicolumn{3}{c}{ LVIS v0.5 Instance Segmentation } \\
\cline { 3 - 5 } & & $\mathrm{AP}_{\text {all }}^{\text {bbox }}$ & $\mathrm{AP}_{50}^{\text {bbox }}$ & $\mathrm{AP}_{75}^{\text {bbox }}$ \\
\hline 1) Random Init & & 22.5 & 34.8 & 23.8 \\
2) IN-sup & $1.28 \mathrm{M}$ & 24.5 & 38.0 & 26.1 \\
3) IN-sup-50\% & $640 \mathrm{~K}$ & $23.7_{-0.8}$ & $36.7_{-1.3}$ & $25.1_{-1.0}$ \\
4) IN-sup-10\% & $128 \mathrm{~K}$ & $20.5_{-4.0}$ & $32.8_{-6.2}$ & $21.7_{-5.2}$ \\
\hline 5) MoCo-IN & $1.28 \mathrm{M}$ & $24.1_{-0.4}$ & $37.4_{-0.6}$ & $25.5_{-0.6}$ \\
6) MoCo-COCO & $118 \mathrm{~K}$ & $23.1_{-1.4}$ & $35.3_{-2.7}$ & $24.9_{-1.2}$ \\
\hline 7) VirTex & $118 \mathrm{~K}$ & $25.4_{+0.9}$ & $39.0_{+1.0}$ & $26.9_{+0.8}$ \\
\hline
\end{tabular}

Table 8: Downstream Evaluation: LVIS v0.5 Instance Segmentation. We compare VirTex with different pretraining methods for LVIS v 0.5 Instance Segmentation. All methods use Mask R-CNN with ResNet-50-FPN backbone. Performance gaps with IN-sup are shown on the side. The trends are similar to LVIS v1.0 Table 3 - VirTex significantly outperforms all baseline methods.

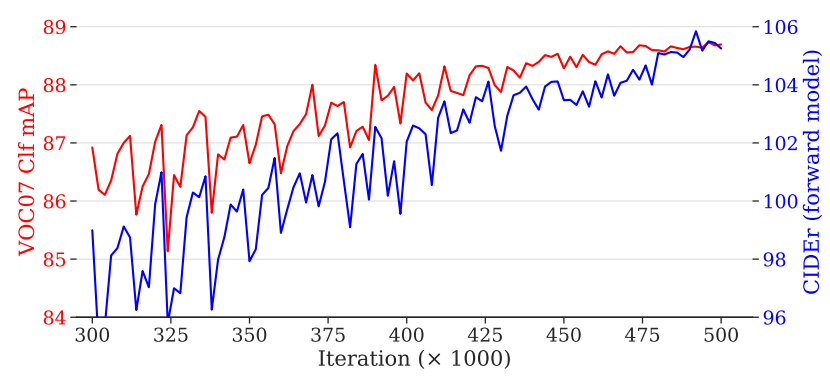

Figure 8: Validation metrics: VOC07 mAP and CIDEr. We compare VOC07 mAP and CIDEr score of VirTex (ResNet-50, $L=1, H=2048$ ) model. We observe that captioning performance has a positive, yet imprecise correlation with downstream performance on vision tasks.

ment in downstream performance. However these are not strongly correlated - the best performing checkpoints according to these metrics occur at different iterations: $496 \mathrm{~K}$ according to VOC07 mAP (88.7), and 492K according to CIDEr (105.8). Hence, we select the best checkpoint based on PASCAL VOC linear classification performance. We use this task as a representative downstream vision task for evaluation due to its speed and simplicity.

\section{Appendix B. Decoder Attention Visualizations for Caption Predictions}

In Figure 9 and Figure 10, we show more qualitative examples showing decoder attention weights overlaid on input images, similar to Section 4.4. All captions are decoded from $L=1, H=512$ VirTex model using beam search. We normalize the attention masks to $[0,1]$ to improve their contrast for better visibility. 

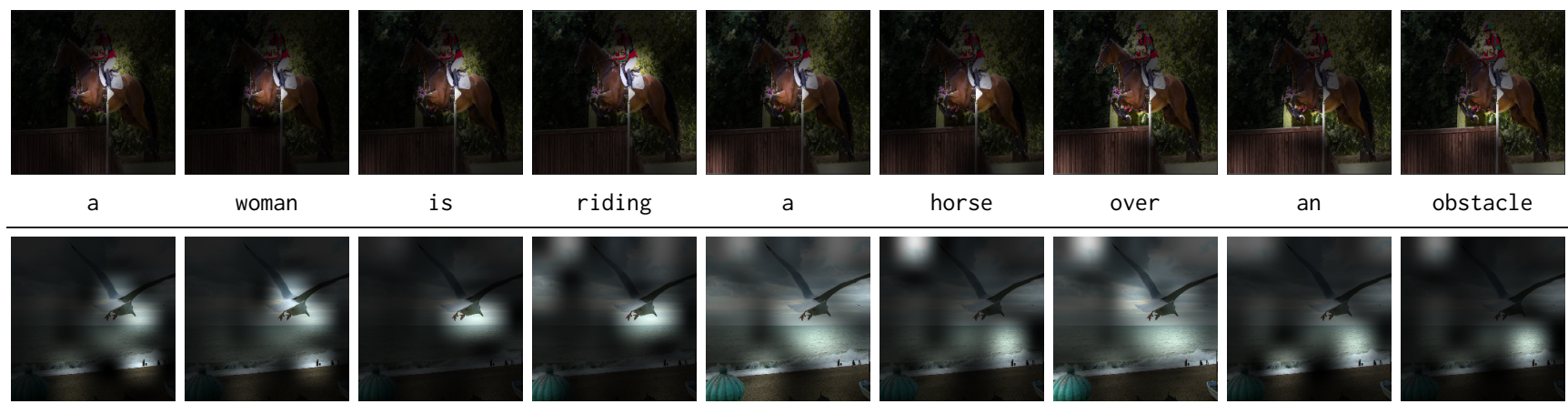

woman

riding

horse

over

obstacle
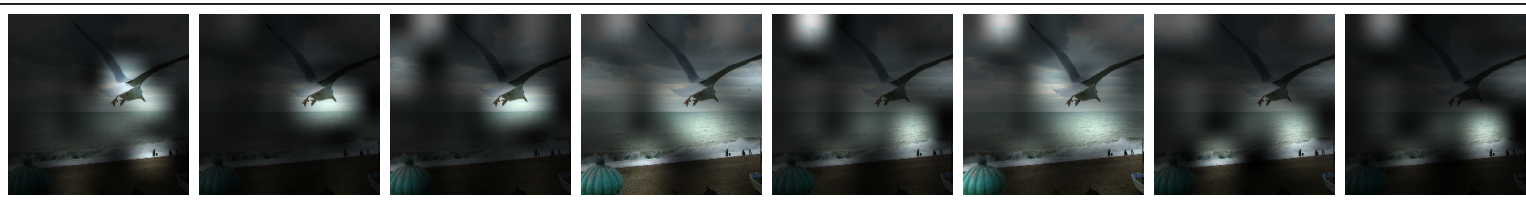

bird

flying

over

air

near

the

ocean
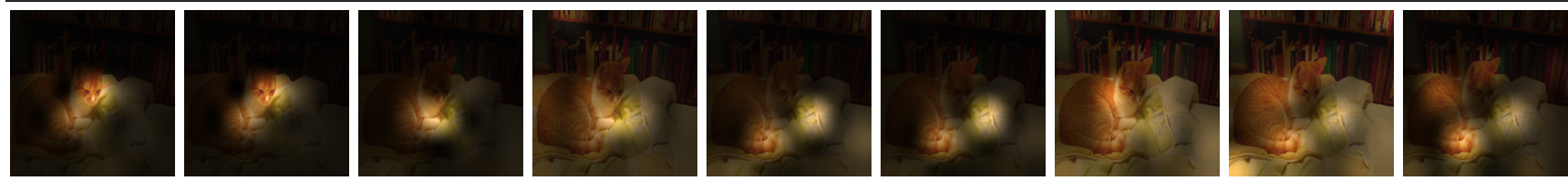

a

laying

a

bed

in

a

bookshelf
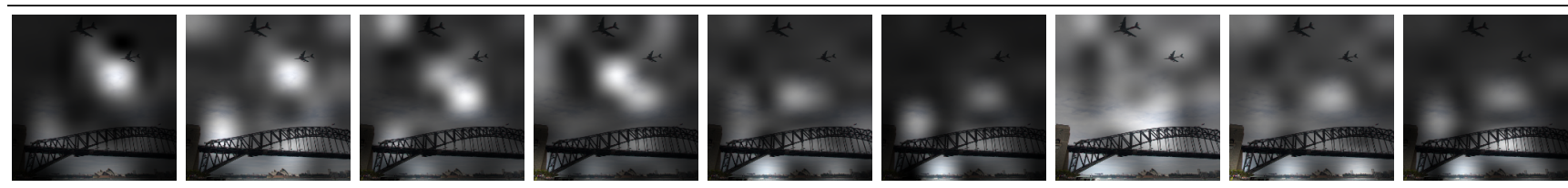

airplane

flying

over

a

body

of

a

river
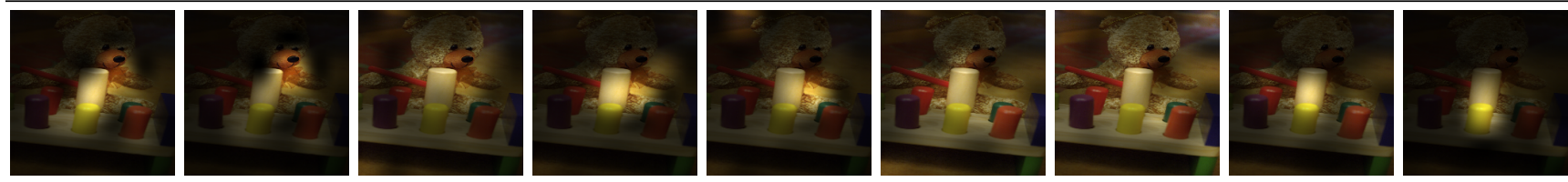

a

teddy

bear

sitting

front

of

orange

juice
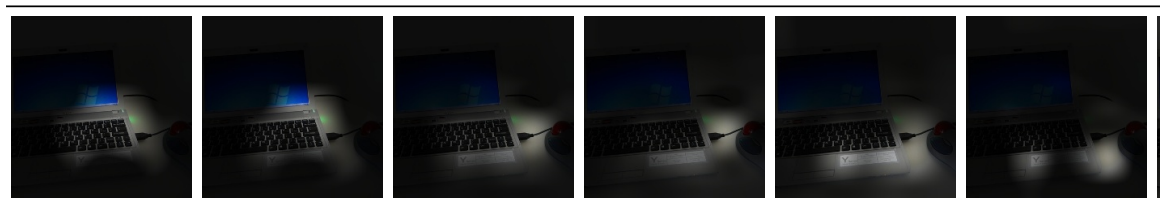

top

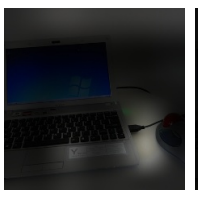

of

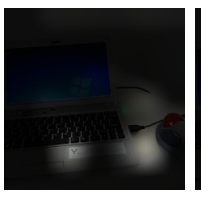

a

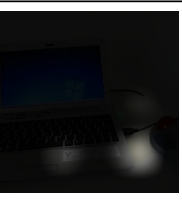

desk

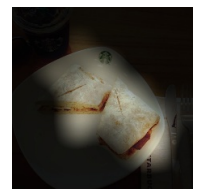

a

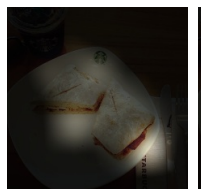

plate

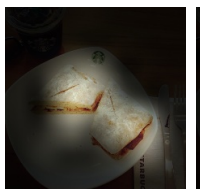

with

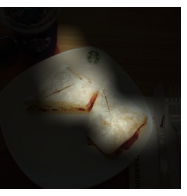

a

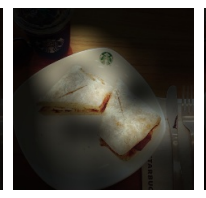

sandwich

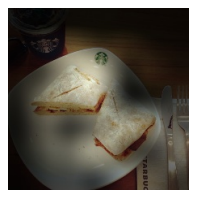

and

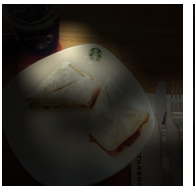

cup

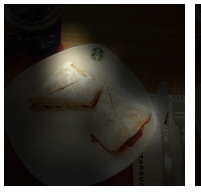

of

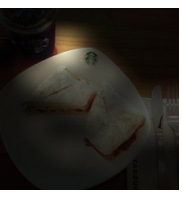

coffee

Figure 9: Attention visualizations per time step for predicted caption. We decode captions from the forward transformer of $L=1, H=512$ VirTex model using beam search. 


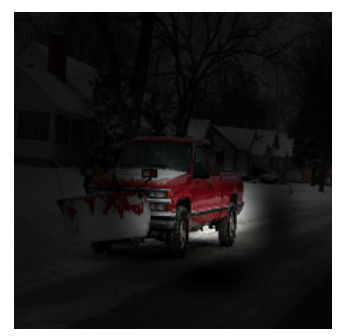

a red truck driving down a snow covered road

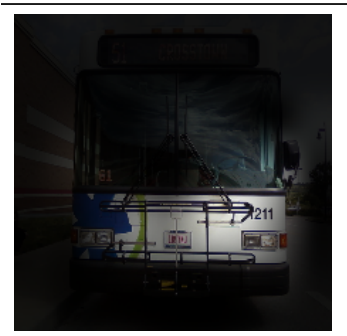

a bus parked at the side of the road

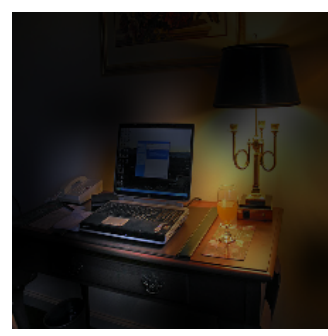

a laptop computer sitting on top of a desk

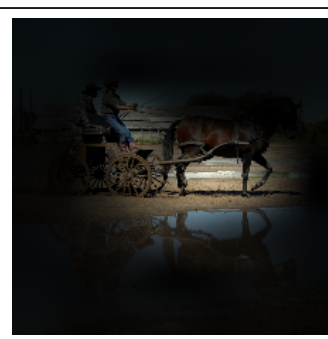

a horse drawn carriage being pulled by two horses

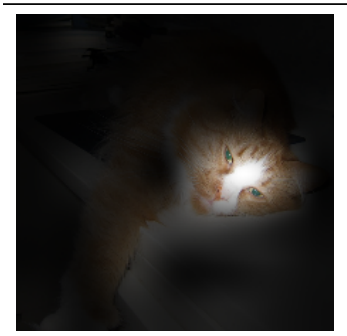

an orange and white cat laying on a desk

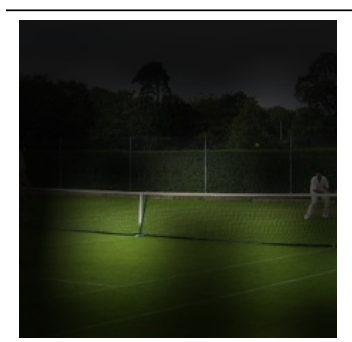

a group of people playing tennis on a tennis court

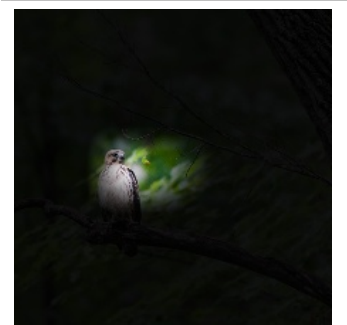

a bird sitting on a branch of a tree

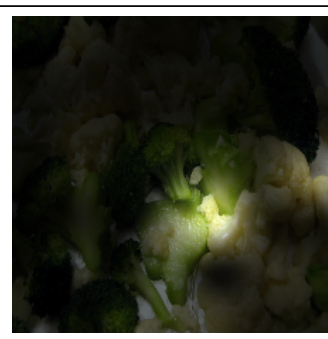

a bowl of broccoli and cauliflower in a lot

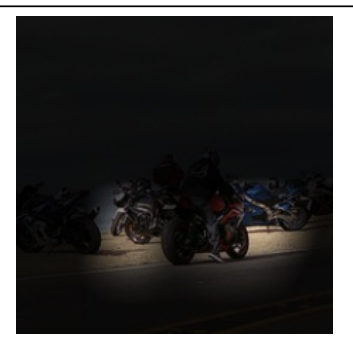

a group of people riding motorcycles down the road

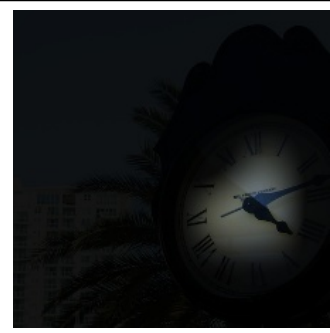

a clock on a building with a clock on it

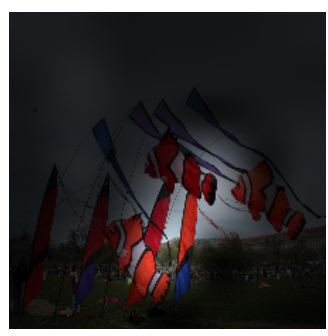

a group of kites being flown in the park

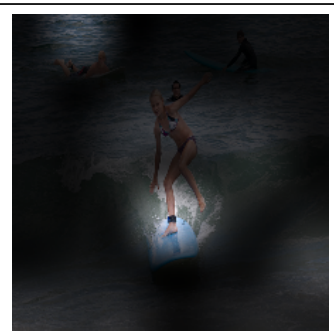

a woman on a wave board in the ocean

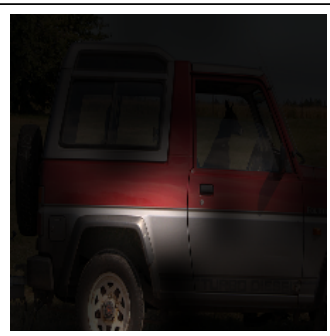

a dog in the back of a red truck

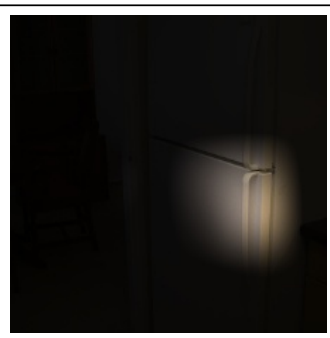

a white refrigerator freezer sitting in a kitchen next to a table

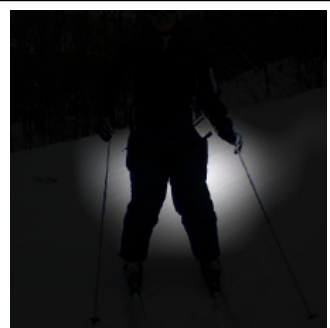

a woman on skis in the side of a snow

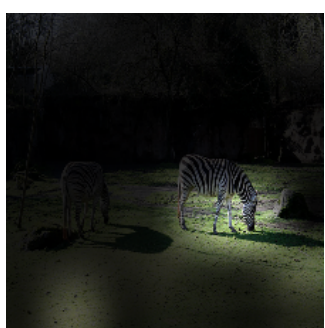

two zebras are grazing in a fenced in area

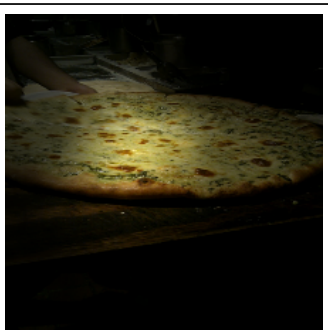

a pizza on a cutting board on a pizza

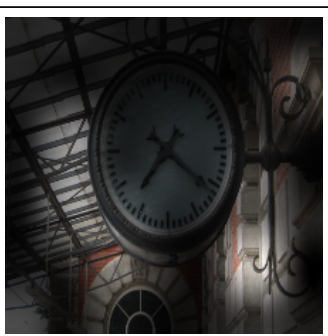

a clock hanging from the a bird perched on top of ceiling in the ceiling

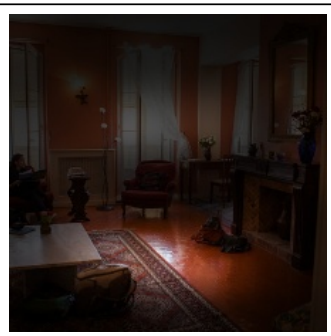

a living room filled with furniture and a fireplace

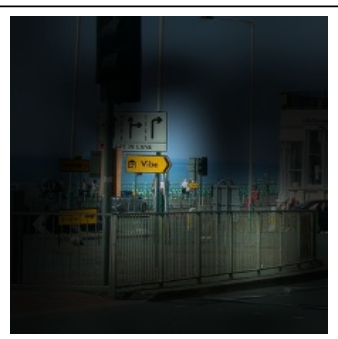

a street sign on it's edge of the road

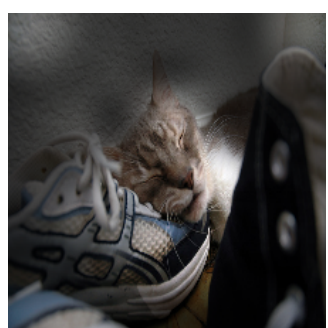

a cat laying on a pair of blue shoes

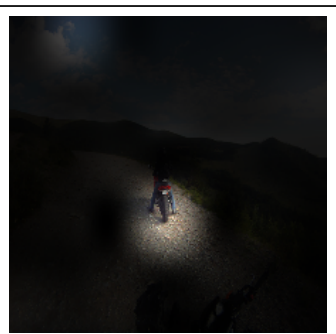

a person riding a motorcycle on a dirt road

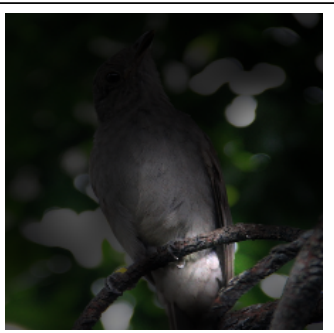
a tree branch

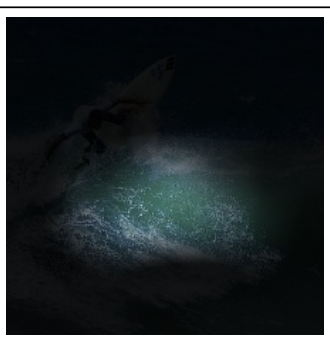

a person on a surfboard riding a wave in the ocean

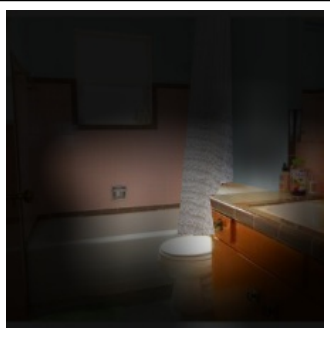

a bathroom with a sink and toilet, toilet

Figure 10: We decode captions from the forward transformer of $L=1, H=512$ VirTex model using beam search. For the highlighted word, we visualize the decoder attention weights overlaid on the input image. 